\title{
Le neuromythe des « styles d'apprentissage » VAK (visuel, auditif, kinesthésique) : une tentative de démystification auprès d'apprentis enseignants franco-ontariens
}

\author{
Luc Rousseau $^{1^{*}}$ et Jeanne Brabant-Beaulieu ${ }^{1}$
}

\begin{abstract}
RÉSUMÉ
Lidée selon laquelle un élève apprend mieux lorsque le contenu pédagogique est présenté dans sa modalité sensorielle préférée (p. ex. présenter des diagrammes ou des images à un élève « visuel») ne repose sur aucun fondement scientifique. Comme d'autres fausses croyances sur le cerveau et l'apprentissage (p. ex. On utilise seulement $10 \%$ de notre cerveau), il s'agit d'un neuromythe. La prévalence internationale d'adhésion au neuromythe des «styles d'apprentissage » VAK (visuel, auditif, kinesthésique) dans le milieu de l'éducation est en moyenne de $88 \%$. Cette fausse croyance semble profondément ancrée dans l'expérience personnelle des enseignants. En effet, dans une recherche récente, après avoir été exposés au savoir scientifique disqualifiant leur utilité pédagogique, $90 \%$ des répondants ont rejeté le bien-fondé conceptuel des styles d'apprentissage, mais un tiers d'entre eux ont indiqué vouloir malgré tout continuer d'employer ce concept dans leur pratique enseignante, dont $89 \%$ en raison de leur expérience personnelle (p. ex. Je l'observe en classe). Les observations anecdotiques sembleraient donc protéger les neuromythes de lassaut du savoir scientifique. Dans la présente étude, nous avons émis l'hypothèse que la création d'une anecdote personnelle disqualifiant lutilité pédagogique des styles d'apprentissage VAK puisse former, avec le savoir scientifique, une alliance suffisamment puissante pour faire contrepoids aux anecdotes déjà vécues par les répondants et déstabiliser cette fausse croyance. Des apprentis enseignants ont réalisé une activité didactique dans laquelle le contenu pédagogique était présenté dans la modalité sensorielle (visuelle ou auditive) correspondant ou non à leur propre «style d'apprentissage » (présumé). Les participants ont ensuite été confrontés à la fois à l'absence de preuves scientifiques et à leurs propres données personnelles disqualifiant lidée d'un meilleur apprentissage lorsque la modalité sensorielle dans laquelle le contenu pédagogique est présenté correspond au «style d'apprentissage » de lapprenant. De $100 \%$ avant lintervention, le pourcentage d'apprentis enseignants ayant indiqué avoir lintention d'utiliser des pratiques pédagogiques inspirées des styles d'apprentissage VAK a décliné à $60 \%$ après lintervention. Ce taux considérable de résistance à lintervention suggère que l'anecdote créée n’avait pas une puissance suffisante pour déstabiliser la fausse croyance. Des pistes de recherche futures sont suggérées pour consolider la nouvelle anecdote, dont notamment une intervention visant à réaliser l'activité didactique auprès d'élèves, de sorte que les participants puissent être témoins de contre-exemples directement issus du milieu éducatif.
\end{abstract}

\footnotetext{
${ }^{1}$ Département de psychologie, Université Laurentienne, Sudbury, ON, Canada

* Correspondance avec l'auteur : lrousseau@laurentienne.ca
}

Pour citer cet article: Rousseau, L. et Brabant-Beaulieu, J. (2020). Le neuromythe des «styles d'apprentissage » VAK (visuel, auditif, kinesthésique) : une tentative de démystification auprès d'apprentis enseignants franco-ontariens. Neuroéducation, 6(1), 65-91.

DOI: https://doi.org/10.24046/neuroed.20200601.37

Reçu le 15 août 2019. Révision reçue le 20 décembre 2019. Accepté le 7 janvier 2020. Publié en ligne le 6 juin 2020.

Neuroéducation, 6(1), 65-91

ISSN: $1929-1833$

Tous droits réservés (C) 2020 - Association pour la recherche en neuroéducation 


\section{Introduction}

La très grande majorité des recherches scientifiques en neuroéducation portent sur le fonctionnement du cerveau de l'apprenant en général. Or, les instances scolaires insistent beaucoup sur l'unicité de chaque élève et sur le devoir des enseignants de répondre aux besoins individuels en matière d'apprentissage. Dans ce contexte, certaines idées fausses, mais qui semblent intuitivement fondées, peuvent exercer un attrait sur le milieu de l'éducation, dont celle de l'existence de «profils» particuliers de fonctionnement cérébral chez les apprenants. En effet, pour répondre aux besoins de ses élèves, un enseignant pourrait être enclin à adapter son enseignement aux élèves « cerveau droit », aux élèves avec un «style d'apprentissage kinesthésiques», ou encore aux élèves dotés d'une « intelligence interpersonnelle ». Or, de tels profils particuliers de fonctionnement cérébral chez les apprenants, non appuyés par la recherche scientifique, sont qualifiés de neuromythes: des fausses croyances sur le cerveau et l'apprentissage (p. ex. Geake, 2008; Howard-Jones, 2014; Masson, 2015; Pasquinelli, 2012; Tardif et Doudin, 2010).

Si les pratiques pédagogiques inspirées des neuromythes traduisent un intérêt croissant du milieu de l'éducation envers les fondements cérébraux de l'apprentissage, leur adoption pourrait toutefois entraîner des effets nuisibles. Ainsi, un élève étiqueté comme un apprenant « visuel » pourrait percevoir sa capacité d'apprentissage comme étant confinée à des contenus pédagogiques uniquement présentés dans cette modalité sensorielle (angl. "pigeon-holing"; Newton, 2015). De plus, une trop vaste adhésion de la profession enseignante aux neuromythes et le recours à des pratiques pédagogiques inspirées de telles fausses croyances pourraient entraîner une perte de temps et de ressources précieuses, qui seraient mieux investies dans l'adoption de pratiques pédagogiques probantes, appuyées par la recherche scientifique.

Dans cet article, nous abordons, dans un premier temps, trois neuromythes populaires dans le milieu de l'éducation. Dans un deuxième temps, nous nous attardons aux enquêtes internationales sur les neuromythes, avec une emphase particulière sur celui des «styles d'apprentissage » VAK (visuel, auditif, kinesthésique). Nous soulevons ensuite, dans un troisième temps, l'influence exercée par les biais cognitifs sur l'adhésion à de telles fausses croyances. Dans un quatrième temps, nous passons en revue des études expérimentales ayant testé l'efficacité d'interventions visant à dissiper les neuromythes. Enfin, dans un cinquième temps, nous proposons une intervention originale visant à démystifier, spécifiquement, le neuromythe des styles d'apprentissage VAK, puis rapportons les résultats d'une étude expérimentale visant à tester l'efficacité de cette intervention auprès d'un échantillon d'apprentis enseignants franco-ontariens inscrits dans un programme de formation initiale à l'enseignement.

\subsection{Trois neuromythes populaires}

Dans cette section, nous abordons trois neuromythes populaires dans le milieu de l'éducation. Il s'agit de fausses croyances sur l'existence de profils particuliers de fonctionnement cérébral chez les apprenants: le neuromythe de la dominance hémisphérique, le neuromythe des intelligences multiples et le neuromythe des styles d'apprentissage VAK.

D'abord, un neuromythe fort répandu dans le milieu de l'éducation est celui de la dominance hémisphérique, selon lequel l'un des deux hémisphères cérébraux serait prédominant dans le processus d'apprentissage. Les expressions « élève cerveau gauche » et «élève cerveau droit» cristallisent cette fausse croyance. Entre autres facteurs, une extrapolation abusive de travaux scientifiques sur la spécialisation fonctionnelle des hémisphères cérébraux pourrait avoir contribué à l'émergence de ce neuromythe (Lindell et Kidd, 2011). Des travaux désormais classiques ont été réalisés dans les années 1960 (p. ex. Gazzaniga, Bogen et Sperry, 1965) chez des patients dont le corps calleux - un faisceau composé de 200 millions de fibres nerveuses connectant les deux hémisphères - a été sectionné chirurgicalement (callosotomie). Dans des conditions de laboratoire très contrôlées, une certaine latéralisation des fonctions cérébrales (notamment les fonctions langagières dans l'hémisphère gauche et les fonctions spatiales dans l'hémisphère droit) se manifeste chez les rares patients dits à cerveau divisé (angl. split brain patients; pour une synthèse, voir Gazzaniga, 2005; Sperry, 1982).

Des travaux plus récents de neuroimagerie par résonance magnétique fonctionnelle ont également démontré que les fonctions langagières sont, chez la plupart des individus «normaux » (et non seulement chez les patients à cerveau divisé), latéralisées dans l'hémisphère gauche (p. ex. Holland et al., 2007). Toutefois, latéralisation et dominance hémisphérique ne sont pas des termes interchangeables. En effet, une étude de neuroimagerie par résonance magnétique fonctionnelle, réalisée par Nielsen et ses collaborateurs (2013) chez 1011 individus « normaux 》 (424 femmes et 587 hommes, dont l'âge variait entre 7 et 29 ans), a confirmé la latéralisation des fonctions langagières dans l'hémisphère gauche et suggéré la latéralisation de fonctions attentionnelles dans l'hémisphère droit. Cependant, ces données de neuroimagerie fonctionnelle n'ont dévoilé aucun patron particulier d'activité corticale démontrant la dominance d'un hémisphère cérébral sur l'autre. Par conséquent, les pratiques pédagogiques adaptées aux élèves « cerveau droit » (soi-disant intuitifs, émotionnels et créatifs) et aux élèves «cerveau gauche » (soi-disant analytiques, logiques et rationnels) ne sont pas appuyées par la recherche scientifique.

Ensuite, un autre neuromythe fort populaire en éducation est celui des intelligences multiples. En effet, certaines théories formulées par des chercheurs universitaires, mais qui n'ont pas surmonté l'épreuve de la validation scientifique, peuvent exercer un attrait sur le milieu de l'éducation, en particulier si ces théories mettent l'emphase sur des différences individuelles sur le plan du fonctionnement cérébral. C'est le 
cas de la théorie des intelligences multiples (Gardner, 1983/1997), qui propose l'existence de formes d'intelligence variées (musicale, corporelle-kinesthésique, visuo-spatiale, logico-mathématique, verbale-linguistique, interpersonnelle, naturaliste...), neurologiquement indépendantes les unes des autres. Tout individu serait doté de ces intelligences, mais à des degrés divers. Selon Visser, Ashton et Vernon (2006), l'attrait exercé par la théorie des intelligences multiples sur le milieu de l'éducation s'explique en partie par la vision «égalitariste» que dégage cette théorie: tous les élèves auraient une chance égale d'être intelligents, mais chacun posséderait sa propre forme d'intelligence. Si un élève éprouve de la difficulté en mathématique, il pourrait néanmoins réussir en littérature, en musique, ou encore en éducation physique.

Or, aussi populaire et séduisante que puisse être la théorie des intelligences multiples dans le contexte des politiques actuelles de «réussite éducative pour tous » et d'inclusion scolaire, ses assises empiriques sont quasi-inexistantes et son auteur s'est dissocié de sa formulation initiale (Gardner, 2016). En plus de la nature même des formes d'intelligence décrites par Gardner (1983/1997), qui s'apparenteraient davantage à des talents, leur indépendance a sérieusement été remise en question (Furnham, 2009; Larivée et Sénéchal, 2012; Visser et al., 2006; Waterhouse, 2006; Willingham, 2004). En raison du manque de preuves scientifiques de leur validité, les intelligences multiples sont considérées par de nombreux auteurs comme un neuromythe (Blanchette Sarrasin, Riopel et Masson, 2019; Dekker, Lee, Howard-Jones et Jolles, 2012; Ferrero, Garaizar et Vadillo, 2016; Geake, 2008; Howard-Jones, 2014; Howard-Jones, Franey, Mashmoushi et Liao, 2009; Ruhaak et Cook, 2018). Par conséquent, les pratiques pédagogiques adaptées aux intelligences multiples des apprenants ne sont pas appuyées par la recherche scientifique.

Enfin, un neuromythe omniprésent dans le milieu de l'éducation est celui de l'existence de styles d'apprentissage, et notamment de styles basés sur les modalités sensorielles visuelle, auditive et kinesthésique (VAK). Selon Masson (2015), deux idées fausses sous-tendent ce neuromythe. Premièrement, il y a l'idée fausse que chaque apprenant possède un cerveau unique, donc possède un style d'apprentissage unique. Or, s'il est vrai qu'à partir des quelque 100 milliards de neurones dont dispose le cerveau humain à la naissance, un réseau unique de connections synaptiques se développe chez un individu, il est faux d'affirmer qu'un tel développement individualise entièrement le cortex, au point de rendre chaque cerveau unique et de l'optimiser pour traiter plus efficacement une information sensorielle spécifique (visuelle, auditive, ou kinesthésique) ${ }^{1}$. En effet, si le cortex est doté d'aires sensorielles anatomiquement distinctes, l'idée que l'une d'entre elles puisse être stimulée de manière isolée et dominer les autres aires sensorielles est incompatible avec les connaissances scientifiques sur l'architecture fonctionnelle du cerveau. Le cortex est en effet constitué d'un ensemble de réseaux neuronaux anatomiquement distribués. Notamment, les aires corticales sensorielles sont interconnectées via des points de passage à haut débit (angl. hub nodes), favorisant le transfert intermodal de l'information (Álvarez-Montero, ReyesSosa, Leyva-Cruz et Fragoza-Padilla, 2019). De plus, des aires corticales associatives dites hétéromodales, communes à tous les cerveaux humains, intègrent les signaux sensoriels unimodaux pour constituer des perceptions unifiées (Calvert, Campbell et Brammer, 2000). Par conséquent, affirmer que le cerveau prédispose un apprenant à un style d'apprentissage unique basé sur une modalité sensorielle donnée relève d'une fausse croyance.

Deuxièmement, selon Masson (2015), il y a l'idée fausse qu'un enseignement adapté au style d'apprentissage préféré d'un apprenant soit, par le fait même, adapté à son profil particulier de fonctionnement cérébral, et donc qu'un tel enseignement optimise son apprentissage. En soi, les préférences personnelles pour recevoir l'enseignement dans l'une ou l'autre modalité sensorielle ne constituent pas un neuromythe. Les préférences personnelles sont ce qu'elles sont : de simples préférences. Toutefois, l'idée selon laquelle un élève apprend mieux lorsque le contenu pédagogique est présenté dans sa modalité sensorielle préférée constitue, à la lumière des connaissances scientifiques actuelles, un neuromythe. En effet, selon l'hypothèse dite d'appariement (angl. matching hypothesis), la qualité de l'apprentissage devrait s'avérer supérieure quand l'enseignement est apparié à la modalité sensorielle dite préférée de l'apprenant (Pashler, McDaniel, Rohrer et Bjork, 2008).

Or, selon une synthèse récente de la littérature scientifique (Rousseau, Gauthier et Caron, 2018), aucune démonstration empirique de la validité de l'hypothèse d'appariement n'est disponible à ce jour, et ce, quelle que soit la méthode scientifique utilisée (devis corrélationnel, devis expérimental, neuroimagerie par résonance magnétique fonctionnelle, stimulation magnétique transcrânienne, enregistrement des mouvements oculaires), quel que soit l'instrument utilisé pour mesurer les styles d'apprentissage VAK et quelle que soit la mesure de rendement (mémorisation, compréhension, jugement de similarité, note finale dans un cours). Trois autres études expérimentales, publiées depuis, ont rejeté l'hypothèse d'appariement (Aslaksen et Lorås, 2019; Cuevas et Dawson, 2018; Rogowsky, Calhoun et Tallal, 2020). De plus, une méta-analyse récente (Aslaksen et Lorås, 2018), réalisée sur un corpus de dix études scientifiques totalisant 13 expériences, a révélé une taille d'effet globale faible et non significative, tant pour un appariement visuel $(g=-0,09)$ que pour un appariement auditif $(g=-0,027)$. Par conséquent, les pratiques pédagogiques adaptées aux styles d'apprentissage VAK (p. ex. visionnement d'images ou de diagrammes pour les élèves «visuels »; écoute de sons ou de paroles pour les élèves «auditifs »; manipulation d'objets pour les élèves «kinesthésiques ») ne sont pas appuyées par la recherche scientifique.

\footnotetext{
${ }^{1}$ De plus, les connections synaptiques ne sont pas permanentes, mais plutôt malléables (plasticité cérébrale) tout au long de la vie.
} 
À titre d'illustration de l'une des nombreuses mises à l'épreuve empiriques de l'hypothèse d'appariement, Cuevas et Dawson (2018) ont d'abord administré à leurs participants un questionnaire pour déterminer si leur style d'apprentissage était davantage à prédominance visuelle ou auditive. Puis, les participants étaient invités à compléter une tâche dans laquelle 20 phrases étaient présentées oralement (p. ex. : La grenouille verte sauta dans la piscine; traduction libre). La moitié des participants ont reçu la directive de visualiser la phrase, puis d'indiquer, sur une échelle de type Likert à cinq niveaux (de $1=$ impossible à imaginer à $5=$ très facile à d'imaginer), leur degré de facilité à former une image mentale claire (angl. vivid) de l'action exécutée dans la phrase. L'autre moitié des participants ont reçu la directive de penser aux sons produits par les mots de la phrase, en répétant la phrase silencieusement, puis d'indiquer, sur une échelle de type Likert à cinq niveaux (de 1 = très difficile à prononcer à 5 = très facile à prononcer), leur degré de facilité à prononcer la phrase. Par la suite, les participants étaient conviés à un test de rappel indicé, consistant à répondre à 20 questions correspondant aux 20 énoncés (p. ex. : La grenouille verte sauta dans quoi?).

Selon l'hypothèse d'appariement, les participants avec un style d'apprentissage prédominant «visuel » devraient obtenir un meilleur score de rappel indicé à la suite d'une tâche de visualisation mentale plutôt qu'à la suite d'une tâche de prononciation, alors qu'un patron inverse de résultats devrait être obtenu par les participants avec un style d'apprentissage prédominant «auditif». Or, aucune interaction croisée GROUPE (《visuel » vs. « auditif ») × TÂCHE (visualisation vs. prononciation) - soit la signature de l' effet d'appariement (Pashler et al., 2008) - ne fut observée. En fait, le score de rappel indicé s'est avéré significativement supérieur à la suite d'une tâche de visualisation mentale (effet principal de TÂCHE), quel que soit le style d'apprentissage prédominant des participants (tel que déterminé par le questionnaire). Cuevas et Dawson (2018) ont conclu que la théorie du double encodage (Paivio, 1971, 1986) était plus apte à expliquer les résultats que le concept de styles d'apprentissage VAK.

Bien que dans la littérature tant scientifique que populaire actuelle, l'expression «style d'apprentissage » soit devenue quasi-synonyme de préférence d'apprentissage VAK, une multitude de modèles de styles d'apprentissage ont été proposés depuis plus de 40 ans (pour une synthèse historique, voir Chevrier, Fortin, Leblanc et Théberge, 2000). En effet, Coffield, Moseley, Hall et Ecclestone (2004a, 2004b) ont répertorié 71 modèles de styles d'apprentissage. Dans la plupart de ces modèles, les styles d'apprentissage sont exprimés sous la forme de dichotomies - à titre d'exemples : style global vs. analytique; style séquentiel vs. simultané; style réflexif vs. actif. Toutefois, après une analyse exhaustive des 13 modèles de styles d'apprentissage jugés les plus influents, Coffield et al. (2004a, 2004b) ont conclu que les qualités conceptuelles et psychométriques de ces modèles ne répondent pas aux standards scientifiques.
En somme, les mises en garde envers l'adhésion aux neuromythes et l'adoption de pratiques pédagogiques inspirées de ces fausses croyances sur le cerveau et l'apprentissage sont nombreuses, tant dans la sphère scientifique (Alferink, 2007; Dembo et Howard, 2007; Doudin, Tardif et Meylan, 2016; Geake, 2008; Kirschner, 2017; Masson, 2015; Newton, 2015; Pasquinelli, 2012; Riener et Willingham, 2010; Rohrer et Pashler, 2012; Rousseau et al., 2018; Scott, 2010; Sharp, Bowker et Byrne, 2008; Stahl, 1999; Willingham, Hughes et Dobolyi, 2015) que dans la sphère publique (Adey et Dillon, 2012; Baillargeon, 2013; De Bruyckere, Kirschner et Hulshof, 2015; Hood et al., 2017; Pasquinelli, 2015). Or, en dépit de telles mises en garde répétées, les enquêtes internationales attestent d'une adhésion massive aux neuromythes dans le milieu de l'éducation.

\subsection{Enquêtes sur la prévalence des neuromythes}

Plusieurs enquêtes internationales sur la prévalence des neuromythes ont été réalisées au cours des dix dernières années, de sorte que des données sont maintenant disponibles pour 14 pays, répartis sur cinq continents. Les échantillons sondés sont surtout composés d'apprentis enseignants (en formation initiale ou spécialisée), d'enseignants en exercice (au niveau préscolaire, primaire ou secondaire), ainsi que d'étudiants et professeurs d'institutions d'enseignement supérieur (au collège ou à l'université). Largement inspirée de l'enquête influente de Dekker et al. (2012), la méthodologie employée dans l'ensemble des enquêtes est relativement homogène. Une série d'énoncés est présentée aux répondants. La moitié des énoncés sont des affirmations générales non contestées sur le cerveau et l'apprentissage (p. ex. L'apprentissage produit des changements dans les connexions entre les neurones), tandis que l'autre moitié des énoncés sont des affirmations faisant référence à des neuromythes (p. ex. On utilise seulement $10 \%$ de notre cerveau). Les répondants indiquent leur degré d'accord avec chaque énoncé, ce qui sert à établir le taux de connaissances en neurosciences, d'une part, et d'adhésion aux neuromythes, d'autre part.

Fait intéressant, la relation entre les connaissances générales non contestées sur le cerveau et l'apprentissage et l'adhésion aux neuromythes semble différer selon que les répondants soient des apprentis enseignants ou des enseignants dans l'exercice de leur profession. Ainsi, plus les apprentis enseignants possèdent de connaissances générales non contestées sur le cerveau et l'apprentissage, moins ils adhèrent aux neuromythes (Howard-Jones et al., 2009; PapadatouPastou, Haliou et Vlachos, 2017). Inversement, plus les enseignants en exercice possèdent de telles connaissances générales, plus ils adhèrent aux neuromythes (Dekker et al., 2012; Ferrero et al., 2016; Gleichgerrcht, Lira Luttges, Salvarezza et Campos, 2015). Ce patron de résultats semble suggérer que l'exposition aux neurosciences puisse servir de «bouclier » contre les neuromythes, du moins chez les étudiants fréquentant des programmes de formation en enseignement. Toutefois, il s'agit d'une relation corrélationnelle qui, nous le 
verrons plus loin, ne résiste pas à la mise à l'épreuve expérimentale.

De plus, toutes les enquêtes sur les neuromythes que nous avons recensées, à une exception près (Hermida, Segretin, García et Lipina, 2016), ont sondé l'adhésion au neuromythe des styles d'apprentissage VAK. L'énoncé le plus fréquemment utilisé est celui de Dekker et al. (2012) : «Les individus apprennent mieux quand ils reçoivent l'information dans leur style d'apprentissage préféré (p. ex. visuel, auditif, kinesthésique) ». Le tableau 1 présente la prévalence du neuromythe des styles d'apprentissage VAK dans divers pays. Tous échantillons confondus $(n=25)$, le pourcentage de répondants adhérant au neuromythe des styles d'apprentissage VAK varie de $58 \%$ à $98 \%$, avec une moyenne de $88 \%$ et un écart-type de 10,6 . Chez les échantillons composés uniquement d'enseignants $(n=11)$, la prévalence moyenne est de $90 \%$ (é.-t. $=7,8$ ). Chez les trois échantillons composés uniquement d'apprentis enseignants en formation initiale, la prévalence est de $97 \%$ (Australie), de $94 \%$ (Grèce) et de $82 \%$ (Royaume-Uni). Enfin, chez les trois échantillons composés uniquement d'apprentis enseignants poursuivant une spécialité, la prévalence est de $93 \%$ à $95 \%$ (biologie; Allemagne) et de 77 \% (éducation spécialisée; États-Unis). La prévalence la plus forte (98\%) a été observée en Turquie, chez un échantillon hétérogène d'apprentis enseignants en formation initiale ou spécialisée (mathématiques ou sciences).

Parmi les neuromythes sondés, celui des styles d'apprentissage VAK enregistre la prévalence la plus élevée chez 14 des 25 échantillons recensés au tableau 1, ce qui témoigne de son ubiquité. Deux autres fausses croyances se disputent la première place : le neuromythe de la dominance hémisphérique (p. ex. «Des différences dans la dominance cérébrale [cerveau gauche, cerveau droit] peuvent aider à expliquer des différences individuelles entre les apprenants »; Dekker et al., 2012, traduction libre) et le neuromythe de l'environnement enrichi (p. ex. «Les environnements riches en stimulations améliorent le cerveau des jeunes enfants »; Ibid., traduction libre).

Par ailleurs, l'évolution de la prévalence d'adhésion aux neuromythes est difficile à établir, aucun échantillon particulier n'ayant fait l'objet d'un suivi longitudinal. Globalement, pour le neuromythe des styles d'apprentissage VAK (voir tableau 1), chez 13 échantillons de répondants sondés entre 2009 et 2016, la prévalence moyenne était de $90 \%$ (é.-t. $=8,9$ ), tandis que chez 12 échantillons de répondants sondés entre 2017 et 2019, elle était de 85 \% (é.t. = 11,4). Cependant, en raison de l'hétérogénéité des pays, des échantillons et des sondages, il est difficile d'établir un profil évolutif. Ainsi, au Royaume-Uni, si la prévalence d'adhésion au neuromythe des styles d'apprentissage VAK était de $93 \%$ en 2012 chez les enseignants (niveaux primaire et secondaire; Dekker et al., 2012), cinq ans plus tard, elle était de $58 \%$ chez les professeurs (collèges et universités; Newton et Miah, 2017). Or, les professeurs présentaient déjà, en 2014, une prévalence moindre (64\%), du moins aux États-Unis (Dandy et Bendersky, 2014). Ces données semblent donc indiquer un taux d'adhésion moindre chez les professeurs, plutôt qu'une tendance vers le déclin de l'adhésion aux neuromythes dans le milieu de l'éducation. L'origine de la disparité enseignants/professeurs mériterait par ailleurs d'être approfondie.

De plus, le neuromythe des styles d'apprentissage VAK inspirerait nombre de pratiques pédagogiques. Au Québec, dans l'enquête réalisée par Blanchette Sarrasin et al. (2019) aux niveaux préscolaire, primaire et secondaire, près des deux tiers $(64,9 \%)$ des enseignants se disant plutôt en accord ou fortement en accord avec l'énoncé du neuromythe des styles d'apprentissage VAK « Les individus apprennent mieux quand ils reçoivent l'information dans leur style d'apprentissage préféré (p. ex. visuel, auditif, kinesthésique) » ont indiqué utiliser régulièrement les styles d'apprentissage VAK dans leur pratique enseignante. Dans des institutions d'enseignement supérieur (collèges et universités), des professeurs britanniques ont indiqué, dans une proportion égale de $33 \%$, avoir déjà évalué les styles d'apprentissage en classe et avoir utilisé, au cours des douze derniers mois, des méthodes visant à adapter leur enseignement aux styles d'apprentissage de leurs étudiants (Newton et Miah, 2017).

\subsection{L'influence des biais cognitifs}

Dans la littérature scientifique, encore peu de travaux ont été réalisés sur l'influence des biais cognitifs sur l'adhésion aux neuromythes. Pourtant, il est permis de soupçonner qu'il s'agit là d'un facteur important à considérer dans les interventions visant à démystifier ces fausses croyances sur le cerveau et l'apprentissage (Blanchette Sarrasin et al., 2019; Pasquinelli, 2012; Riener et Willingham, 2010).

En effet, l'existence de styles d'apprentissage basés sur la modalité sensorielle préférée d'un élève est une idée intuitivement attrayante. $\mathrm{Si}$ de telles intuitions sont confirmées par des observations anecdotiques, elles peuvent se consolider. Selon Riener et Willingham (2010), le biais de confirmation, la tendance à privilégier les informations qui confortent nos croyances ou idées préconçues au détriment des informations qui les contredisent (Risen et Gilovich, 2007), contribue au maintien du neuromythe des styles d'apprentissage VAK. Ainsi, une enseignante qui observe chez certains élèves une meilleure compréhension d'un concept, après avoir présenté un schéma illustratif, pourrait être amenée à conclure que ces élèves apprennent mieux lorsque le contenu pédagogique est présenté dans la modalité sensorielle qui correspond à leur propre «style d'apprentissage ». Cependant, tel que mentionné par Riener et Willingham (2010) les situations qui contredisent nos intuitions sont peu considérées. Ainsi, l'élève dite auditive qui excelle en musique apprend-elle mieux la géographie en écoutant plutôt qu'en regardant la leçon? 
Tableau 1. Prévalence du neuromythe des styles d'apprentissage VAK dans divers pays.

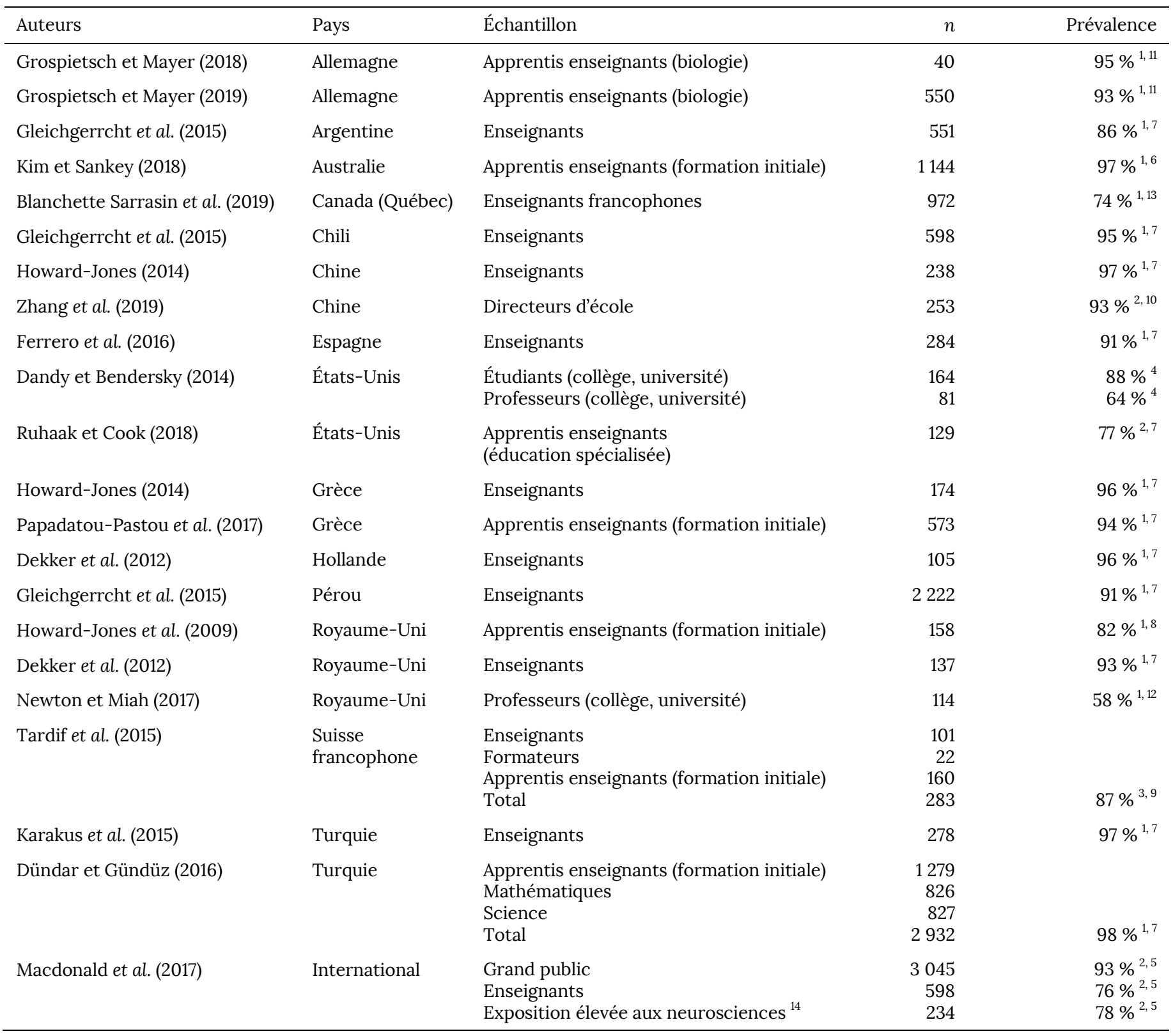

${ }^{1}$ Énoncé (traduction libre): Les individus apprennent mieux quand ils reçoivent l'information dans leur style d'apprentissage préféré (p. ex. visuel, auditif, kinesthésique).

2 Énoncé (traduction libre): Les individus apprennent mieux quand ils reçoivent linformation dans leur style d'apprentissage préféré.

3. Énoncé: Une approche pédagogique basée sur la distinction entre les élèves visuels et auditifs favorise l'apprentissage.

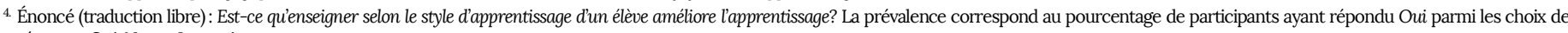
réponses Oui, Non et Incertain.

5. La prévalence correspond au pourcentage de participants ayant indiqué Vrai parmi les choix de réponses Vrai et Faux.

6. La prévalence correspond au pourcentage de participants ayant indiqué Correct parmi les choix de réponses Correct et Incorrect.

7. La prévalence correspond au pourcentage de participants ayant indiqué Correct parmi les choix de réponses Correct, Incorrect et Ne sais pas.

8. La prévalence correspond au pourcentage de participants ayant indiqué En accord parmi les choix de réponses En accord, En désaccord et Ne sais pas.

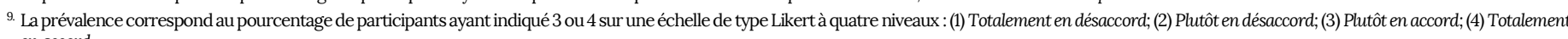
en accord.

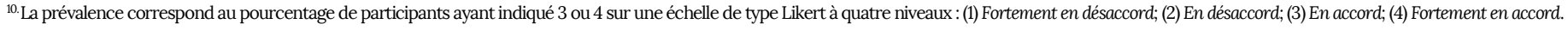

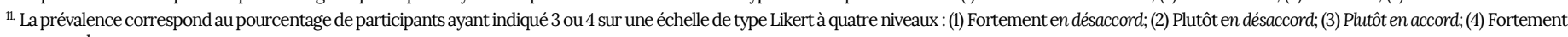
en accord.

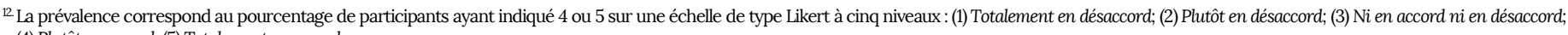
(4) Plutôt en accord; (5) Totalement en accord.

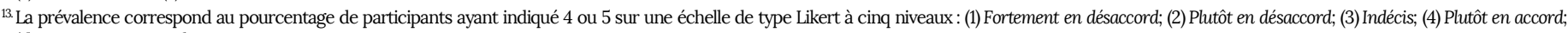
(5) Fortement en accord.

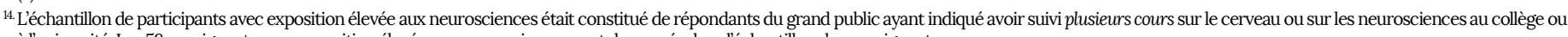
à luniversité. Les 58 enseignants avec exposition élevée aux neurosciences sont demeurés dans léchantillon des enseignants. 
Dans l'étude de Blanchette Sarrasin et al. (2019), $42 \%$ des enseignants québécois adhérant au neuromythe des styles d'apprentissage VAK ont coché Ça m'apparaît logique (intuition) et $62 \%$ Je l'ai observé dans ma pratique (observation anecdotique), parmi les éléments ayant mené à leur niveau d'accord. Pour quatre des cinq neuromythes populaires sondés par Blanchette Sarrasin et al. (2019), l'un de ces deux éléments (intuition ou anecdote) a été coché par les adhérents dans une proportion plus grande que tout autre élément proposé pour justifier leur niveau d'accord (livres et journaux, médias, entourage, formations). Ce résultat suggère que les biais cognitifs, et en particulier ici le biais de confirmation (la tendance à privilégier les observations anecdotiques qui confirment nos intuitions) joue un rôle de premier plan dans l'adhésion aux neuromythes.

Dans l'étude d'Hermida et al. (2016), le neuromythe de la sousutilisation du potentiel cérébral (On utilise seulement $10 \%$ de notre cerveau; voir Larivée, Baribeau et Pflieger, 2008) avait la prévalence la plus élevée : $40 \%$ des enseignants argentins y souscrivaient. Pour justifier leur adhésion à ce neuromythe, les enseignants interviewés par Hermida et al. (2016) ont notamment mentionné que les individus très intelligents, les enfants surdoués, ou encore les personnes capables de perception extra-sensorielle, utilisent $100 \%$ de leur cerveau. La nature simpliste des arguments évoqués témoigne de l'influence possible du biais de simplicité (Feldman, 2016) sur l'adhésion aux neuromythes. Aux théories élaborées et nuancées sur le processus d'apprentissage semblent se substituer des théories simples et tranchées (angl. "all or none" theorising; Howard-Jones et al., 2009). En effet, une conception simpliste du fonctionnement cérébral, à l'origine de plusieurs neuromythes, peut laisser présager des solutions faciles (angl. quick fix) pour l'apprentissage scolaire. Ainsi, sous l'influence du biais de simplicité, il pourrait sembler plus facile à un enseignant de diviser sa classe en deux profils d'apprenants : les élèves «cerveau gauche » et les élèves « cerveau droit » (neuromythe de la dominance hémisphérique).

\subsection{Les interventions}

Les enquêtes internationales ont désormais suffisamment circonscrit l'ampleur du phénomène de la croyance aux neuromythes pour que l'on puisse envisager des tentatives d'interventions pratiques dans le milieu de l'éducation. D'entrée de jeu, comme certains neuromythes constitueraient des distorsions, extrapolations ou simplifications abusives de savoirs neuroscientifiques (Pasquinelli, 2012), exposer les apprentis enseignants aux neurosciences semblerait - du moins à première vue - une stratégie prometteuse pour dissiper les neuromythes. Rappelons que dans certaines enquêtes réalisées auprès d'apprentis enseignants, plus les répondants possédaient de connaissances générales non contestées sur le cerveau et l'apprentissage, moins ils adhéraient aux neuromythes (HowardJones et al., 2009; Papadatou-Pastou et al., 2017).
Or, si le fait d'avoir suivi plusieurs cours en neurosciences aux études supérieures est un facteur prédictif de la nonadhésion aux neuromythes chez le grand public (Macdonald et al., 2017), la taille d'effet est, de l'avis même des auteurs, modeste $\left(\eta^{2} \text { partiel }=0,035\right)^{2}$. De fait, les répondants ayant suivi plusieurs cours en neurosciences n'ont adhéré, en moyenne, qu'à 1,3 neuromythes de moins (sur un total de sept) que les répondants n'ayant suivi aucun cours en neurosciences. D'ailleurs, deux études expérimentales récentes, qui seront maintenant abordées, confirment qu'une simple exposition aux neurosciences s'avère insuffisante pour susciter une baisse de croyance aux neuromythes.

Im, Cho, Dubinsky et Varma (2018) ont introduit un cours de psychologie de l'éducation auprès d'un échantillon expérimental $(n=50)$ d'apprentis enseignants sud-coréens. Un échantillon d'apprentis enseignants de la même cohorte $(n=49)$, non exposés au cours de psychologie de l'éducation, servait de groupe témoin. Le cours était d'une durée d'un trimestre. Le manuel utilisé dans ce cours abordait la controverse entourant certaines pratiques pédagogiques faussement «basées sur le cerveau » et réfutait deux fausses croyances populaires: le neuromythe de la dominance hémisphérique (cerveau gauche/cerveaux droit) et le neuromythe des périodes critiques de développement (p. ex. « Il y a des périodes critiques au cours de l'enfance, après lesquelles certaines choses ne peuvent plus être apprises »; Dekker et al., 2012, traduction libre). Les deux groupes de répondants ont rempli un sondage à deux occasions: au début et à la fin du trimestre. Ce sondage était composé, en ordre aléatoire, de 28 énoncés généraux faisant référence à des connaissances non contestées sur le cerveau et l'apprentissage et de 31 énoncés faisant référence à des neuromythes. Pour chaque énoncé, les participants répondaient oui, non, ou je ne sais pas. Le score de connaissances en neurosciences était établi par le nombre de réponses correctes aux 59 énoncés (oui aux 28 énoncés généraux et non aux 31 énoncés faisant référence à des neuromythes). En retour, le score d'adhésion aux neuromythes était établi par le nombre de réponses incorrectes (oui) aux 31 énoncés faisant référence à des neuromythes.

Im et al. (2018) ont observé un gain significatif, chez le groupe expérimental, de connaissances en neurosciences : leur score post-intervention aux énoncés généraux non contestés sur le cerveau et l'apprentissage $(M=33,60)$ s'est accru par rapport à leur score pré-intervention $(M=28,62)$. Chez le groupe témoin, le score de connaissances en neurosciences est resté inchangé au bout de trois mois ( $M$ pré-test $=27,18$; $M$ posttest $=26,71)$. Cependant, aucun changement n'a été observé dans le score d'adhésion aux neuromythes à la suite de l'intervention. Au départ, les deux groupes ne présentaient pas un niveau de base équivalent, le groupe expérimental adhérant à plus de neuromythes que le groupe témoin. Or, en

\footnotetext{
${ }^{2}$ Une fois converti, l'indice de puissance $\eta^{2}$ partiel $=0,035$ correspond à un $d$ de Cohen $=0,38$ (taille d'effet faible).
} 
dépit de ce biais d'échantillonnage ${ }^{3}$, seul un effet principal significatif de GROUPE a été observé $\left(M_{\text {exp. }}=11,24\right.$ vs. $\left.M_{\text {témoin }}=8,16\right)$, l'interaction GROUPE $\times$ TEMPS (pré- vs. post-test) ne s'étant pas avérée significative. En effet, un examen de la figure $3 b$ de Im et al. (2018) suggère que le score moyen d'adhésion aux neuromythes est demeuré stable chez les deux groupes, n'ayant ni augmenté, ni diminué. En somme, suivre un cours de psychologie de l'éducation semble augmenter les connaissances en neurosciences, sans pour autant réduire l'adhésion aux neuromythes. Pourquoi des connaissances accrues en neurosciences seraient-elles inefficaces pour démystifier les neuromythes?

Selon Grospietsch et Mayer (2018), puisque les fausses croyances sont profondément ancrées dans l'expérience personnelle, les interventions visant à dissiper les neuromythes doivent chercher à atteindre en profondeur les systèmes de croyance, ce que la simple exposition aux neurosciences ne pourrait accomplir qu'implicitement. Une confrontation plus explicite des neuromythes au savoir scientifique serait requise. Ces auteurs se sont inspirés de la littérature sur le changement conceptuel pour proposer une intervention en deux volets. Premièrement, afin que les notions apprises dans un cours de neurosciences cognitives de l'apprentissage soient intégrées en profondeur dans l'expérience personnelle (c'est-à-dire le même niveau d'intégration que les neuromythes), les participants ont été invités à faire des exercices pratiques en classe (p. ex. le traçage d'une forme étoilée en inversion miroir, pour réaliser personnellement que l'apprentissage procédural exige de la pratique).

Deuxièmement, à intervalles réguliers pendant le trimestre, des textes de changement conceptuel étaient remis aux participants comme devoirs à réaliser à la maison. Contrairement aux textes de réfutation au sens strict du terme, les textes de changement conceptuel sont interactifs : une réflexion personnelle est faite, avant et après la lecture du texte de réfutation. La première page du texte de changement conceptuel élaboré par Grospietsch et Mayer (2018) contenait un énoncé faisant référence à un neuromythe (à titre d'exemple: «Les individus apprennent mieux quand ils reçoivent l'information dans leur style d'apprentissage préféré [p. ex. visuel, auditif, kinesthésique] »), suivi d'un espace libre pour faire une réflexion personnelle, c'est-à-dire prendre position sur l'énoncé. Cette première réflexion était suivie par un texte de réfutation de quelques pages, dans lequel des arguments scientifiques étaient soulevés, un à un, pour disqualifier la fausse croyance. En particulier, les contradictions entre le savoir scientifique et le neuromythe étaient explicitement présentées, dans un langage accessible, en évitant le jargon et en utilisant des illustrations de la vie de tous les jours. À titre d'exemple, l'un des cinq arguments utilisés pour contrer le neuromythe des styles d'apprentissage VAK était l'absence d'appuis

\footnotetext{
${ }^{3}$ En effet, puisque le groupe expérimental présentait un taux d'adhésion plus élevé aux neuromythes au pré-test, ce groupe avait, au départ, une plus grande probabilité que le groupe témoin de présenter une diminution de ce taux au post-test, indépendamment de l'intervention.
}

empiriques, dans la littérature scientifique, envers l'hypothèse d'appariement. Les concepts scientifiques pertinents étaient rehaussés par des caractères gras et les arguments rendus plus mémorables à l'aide de sous-titres. Ainsi, le sous-titre «Les tests de styles d'apprentissage sont invalides » (traduction libre) précédait l'argumentation scientifique basée sur les faibles qualités psychométriques des instruments de mesure des styles d'apprentissage VAK. Le texte était suivi de références scientifiques et de suggestions de lectures. Selon Grospietsch et Mayer (2018), la confrontation d'une fausse croyance personnelle au savoir scientifique, après une réflexion personnelle, est susceptible de créer, chez le participant, un « conflit cognitif pouvant servir à initier un changement conceptuel » (p. 5; traduction libre). En ce sens, la dernière page du texte de changement contextuel reprenait l'énoncé faisant référence au neuromythe, suivi d'un espace libre pour faire une seconde réflexion personnelle, c'est-à-dire prendre à nouveau position sur l'énoncé, à la lumière de l'information scientifique présentée.

Grospietsch et Mayer (2018) ont d'abord administré à un échantillon de 57 apprentis enseignants allemands en formation à l'enseignement spécialisé (biologie) un sondage pré-expérimental contenant 11 énoncés faisant référence à des neuromythes et 11 énoncés faisant référence à des connaissances générales non contestées sur le cerveau et l'apprentissage. Puis, l'échantillon a été divisé en deux groupes: un groupe expérimental $(n=40)$ et un groupe témoin $(n=17)$. Les deux groupes de participants ont suivi un cours de neurosciences cognitives de l'apprentissage, d'une durée de 14 semaines, à raison de 90 minutes par semaine. Lors des leçons, les participants des deux groupes ont été invités à réaliser des exercices pratiques en classe. Cependant, seuls les participants du groupe expérimental ont reçu des textes de changement conceptuel. Neuf textes de changement conceptuel ont été distribués pendant le trimestre : sept portant sur des énoncés faisant référence à des neuromythes et deux portant sur des énoncés faisant référence à des connaissances non contestées en neurosciences. En contrepartie, les participants du groupe témoin ont réalisé neuf devoirs de réflexion : après avoir lu des informations sommaires sur des concepts abordés dans le cours, ils devaient faire une réflexion personnelle sur ce que le cours leur avait apporté sur le plan personnel. Certaines de ces informations pouvaient les inciter - toutefois de manière implicite, selon les auteurs - à interroger leurs propres fausses croyances sur le cerveau et l'apprentissage.

Les résultats de l'intervention réalisée par Grospietsch et Mayer (2018) sont encourageants : à la suite de l'intervention, le taux d'adhésion aux 11 neuromythes sondés au pré-test a diminué davantage chez le groupe expérimental que chez le groupe témoin au post-test. L'ANOVA a révélé une 
interaction significative GROUPE $\times$ TEMPS (pré- vs. post-test), avec une taille d'effet très forte $\left(\eta^{2} \text { partiel }=0,524\right)^{4}$. Les cotes moyennes suivantes sont des approximations basées sur la figure 1 de Grospietsch et Mayer (2018). De 3,0 sur une échelle de type Likert à quatre niveaux ( 1 = fortement en désaccord; 2 = plutôt en désaccord; 3 = plutôt en accord; 4 = fortement en accord) avant le cours, la cote moyenne d'accord avec les énoncés faisant référence aux 11 neuromythes a décliné à 2,0 après le cours chez le groupe expérimental. Chez le groupe témoin, la cote moyenne d'accord est demeurée relativement stable à 3,0. Par conséquent, la simple exposition à un cours de neurosciences cognitives de l'apprentissage (groupe témoin) n'a pas été suffisante pour susciter une baisse de croyance aux neuromythes. Les auteurs ont aussi rapporté les pourcentages d'adhésion (cotes 3 et 4 combinées sur l'échelle) aux sept neuromythes ayant fait l'objet d'un texte de changement conceptuel (groupe expérimental). Le pourcentage moyen d'adhésion à ces sept neuromythes est passé de $74 \%$ (pré-test) à $22 \%$ (post-test). Pour le neuromythe des styles d'apprentissage VAK en particulier, le pourcentage moyen d'adhésion est passé de $93 \%$ (pré-test) à $38 \%$ (post-test). Des tests de Wilcoxon ont été réalisés séparément pour chacun des sept neuromythes. Ces tests se sont tous avérés significatifs, dont quatre sur sept (y compris pour le neuromythe des styles d'apprentissage VAK) présentant un indice de puissance $(r)$ allant de 0,538 à $0,590^{5}$.

Pour récapituler, la simple exposition aux neurosciences ne semble pas suffire pour susciter une baisse de croyance envers les neuromythes (Im et al., 2018, ainsi que le groupe témoin de Grospietsch et Mayer, 2018). Cependant, il semble qu'une intervention composée d'une exposition aux neurosciences et de textes de changement conceptuel (groupe expérimental de Grospietsch et Mayer, 2018) puisse susciter une adhésion moindre aux neuromythes. Cependant, il demeure possible que cette baisse d'adhésion aux neuromythes, observée par Grospietsch et Mayer (2018) chez le groupe expérimental, relève davantage d'un effet de désirabilité sociale que d'une réelle baisse de croyance. En effet, dans l'étude qui sera maintenant abordée (Newton et Miah, 2017), une baisse d'adhésion au neuromythe des styles d'apprentissage a été observée à la suite de l'intervention. Toutefois, Newton et Miah (2017) ont attribué cette baisse d'adhésion non pas à une réelle baisse de croyance, mais plutôt à un effet de désirabilité sociale, puisqu'un tiers de leurs participants ont indiqué, à la suite de l'intervention, avoir toujours l'intention de tenir compte du concept de styles d'apprentissage dans leur pratique enseignante. Grospietsch et Mayer (2018) n'ayant pas sondé les intentions pratiques de leurs participants à la suite de l'intervention, on

\footnotetext{
${ }^{4}$ Une fois converti, lindice de puissance $\eta^{2}$ partiel $=0,524$ correspond à un $d$ de Cohen = 1,51 (taille d'effet très forte).

${ }^{5}$ Une fois converti, l'indice de puissance $r=0,538$ correspond à un $d$ de Cohen $=1,28$ (taille d'effet très forte), tandis que l'indice de puissance $r=0,590$ correspond à un $d$ de Cohen $=1,46$ (également une taille d'effet très forte).
}

ne peut conclure formellement en une baisse réelle de croyance envers les neuromythes ciblés dans leur étude.

La recherche de Newton et Miah (2017) misait, pour dissiper les neuromythes, non seulement sur la confrontation au manque de preuves scientifiques pour les appuyer, mais également sur la sensibilisation aux torts que peuvent causer les pratiques pédagogiques inspirées de ces fausses croyances. Ces chercheurs ont réalisé une intervention auprès d'un échantillon de professeurs britanniques $(n=114)$ œuvrant dans des institutions d'enseignement supérieur (collèges ou universités). L'intervention était entièrement réalisée en ligne. De manière fort intéressante, non seulement l'adhésion au neuromythe des styles d'apprentissage a été sondée chez les répondants, mais également leur intention d'utiliser ce concept dans leur pratique enseignante. De prime abord, $58 \%$ des répondants ont affirmé être plutôt d'accord ou totalement d'accord avec l'énoncé de croyance envers le neuromythe des styles d'apprentissage : «Les individus apprennent mieux lorsqu'ils reçoivent l'information dans leur style d'apprentissage préféré (p. ex, visuel, auditif, kinesthésique) ». Leur degré d'accord était encore plus élevé (64\%) avec l'énoncé pratique «J'essaie d'organiser mon enseignement pour accommoder les différents styles d'apprentissage de mes élèves (p. ex. visuel, auditif, assimilateur/convergent) $»^{6}$.

Par la suite, Newton et Miah (2017) ont informé les répondants qu'il n'existait aucune preuve scientifique pour appuyer l'idée selon laquelle adapter l'enseignement aux styles d'apprentissage des élèves améliore l'apprentissage. Cette étape a été suivie par un nouveau sondage, dans lequel les participants devaient indiquer leur niveau d'accord avec des énoncés soulevant des torts que peut poser la prise en compte des styles d'apprentissage dans la pratique enseignante. Neuf répondants sur dix (90 \%) ont alors indiqué être d'accord avec l'énoncé « Nous ne devrions pas utiliser les styles d'apprentissage parce qu'ils sont conceptuellement erronés; cela ne tient pas compte de la complexité du processus d'apprentissage », tandis que $61 \%$ se sont dits d'accord avec l'énoncé « Il s'agit d'une perte de ressources qui devraient être investies ailleurs » (traductions libres). De tels pourcentages laissaient présager que les professeurs allaient être enclins à réviser leurs pratiques pédagogiques inspirées des styles d'apprentissage. Or, de manière plutôt paradoxale, $32 \%$ des répondants ont indiqué être d'accord avec l'énoncé «À la lumière de l'information présentée, je prévois tenir compte des styles d'apprentissage de mes élèves dans mon enseignement » (traduction libre). C'est donc dire qu'en dépit d'un rejet massif (90\%) du bien-fondé conceptuel des styles
${ }^{6}$ À noter que Newton et Miah (2017) ont inclus, parmi les exemples de
styles d'apprentissage fournis dans certains de leurs énoncés, non
seulement les styles d'apprentissage VAK, mais aussi des styles
d'apprentissage proposés dans le modèle de Kolb (1976). Ce modèle
combine deux dimensions bipolaires (concret-abstrait; action-réflexion)
pour proposer quatre styles d'apprentissage : convergent (abstrait actif),
divergent (concret réflexif), assimilateur (abstrait réflexif) et
accommodateur (concret actif). 
d'apprentissage à la suite de l'intervention, près du tiers des professeurs avaient toujours l'intention d'utiliser ce concept dans leur pratique enseignante.

Newton et Miah (2017) ont attribué la volte-face de ce tiers de répondants (c'est-à-dire le maintien de l'intention de tenir compte des styles d'apprentissage en classe malgré la reconnaissance de l'inutilité pédagogique de ce concept) à deux facteurs. D'une part, les auteurs ont évoqué un effet de désirabilité sociale pour expliquer l'adhésion massive des répondants à des énoncés tels que « Nous ne devrions pas utiliser les styles d'apprentissage parce qu'ils sont conceptuellement erronés; cela ne tient pas compte de la complexité du processus d'apprentissage ». Les répondants auraient rejeté l'utilité pédagogique des styles d'apprentissage pour répondre aux attentes des chercheurs, plutôt qu'en raison d'une réelle baisse de croyance. D'autre part, Newton et Miah (2017) ont évoqué, pour expliquer l'intention d'un tiers des répondant de continuer de tenir compte des styles d'apprentissage en classe, un possible effet de retour de flamme (angl. backfire effect), qui survient quand une tentative de démystification produit un effet antagoniste : l'amplification d'une fausse croyance (Nyhan et Reifler, 2010). Ce phénomène est conçu comme une réaction à forte charge émotive. À la suite de leur intervention, Newton et Miah (2017) ont recueilli les commentaires écrits de leurs répondants. Certains répondants ont affirmé que le sondage manquait de partialité, qu'il affichait un biais à l'encontre des styles d'apprentissage et que certaines questions étaient tendancieuses (angl. leading), des commentaires qui semblent laisser transparaître une forte réaction émotive. En somme, en dépit d'une intervention qui semble efficace (rappelons que $90 \%$ des répondants ont rejeté le bien-fondé conceptuel des styles d'apprentissage), une telle intervention pourrait, au contraire, avoir contribué à la consolidation de ce neuromythe chez les participants.

De plus, fait particulièrement intéressant, parmi les $32 \%$ de répondants qui avaient toujours l'intention d'adapter leur enseignement aux styles d'apprentissage de leurs élèves, $89 \%$ ont indiqué être d'accord avec l'énoncé «En dépit du fait qu'il n'existe aucune preuve scientifique de leur existence, mon expérience m'a appris qu'utiliser les styles d'apprentissage est bénéfique pour mes élèves » (traduction libre; Newton et Miah, 2017). Les observations anecdotiques des enseignants dans l'exercice de leur profession semblent donc dicter l'adoption de pratiques pédagogiques dans une plus grande mesure que le savoir issu de la recherche scientifique. Pourquoi les anecdotes personnelles semblentelles déployer une telle puissance face à la confrontation au

\footnotetext{
${ }^{7}$ Brain Gym ${ }^{\circledR}$ est un programme éducatif commercial (Dennison et Dennison, 1994) implanté dans plus de 80 pays (Hyatt, 2007) et qui propose une gamme d'exercices de coordination perceptivo-motrice pour améliorer les fonctions cognitives (p. ex., concentration, mémoire) et favoriser l'apprentissage (p. ex., lecture, écriture, mathématique; Stephenson, 2009). Or, les prétentions pédagogiques de ce programme reposent, selon Hyatt (2007) et Stephenson (2009), sur une conception simpliste du fonctionnement cérébral. Malgré la popularité de ce programme éducatif, jusqu'à présent, aucune étude scientifique n'a démontré que l'usage de Brain Gym ${ }^{\circledR}$ améliore les capacités cognitives ou
}

savoir scientifique? Pasquinelli (2012) offre quelques pistes intéressantes de réflexion à cet égard.

Pour illustrer la forte résistance des neuromythes à la confrontation au savoir scientifique, Pasquinelli (2012) a utilisé l'exemple suivant : «Un enseignant qui adopte Brain Gym $^{\circledR}$ en classe dispose d'anecdotes personnelles riches en émotions, autrement plus mémorables que les statistiques négatives issues d'une méta-analyse scientifique » (p.93; traduction libre) $)^{7}$. L'auteure a évoqué les biais cognitifs comme des mécanismes psychologiques permettant aux anecdotes personnelles de protéger les fausses croyances de l'assaut du savoir scientifique. Notamment, Pasquinelli (2012) a évoqué l'heuristique de disponibilité (angl. availability heuristic), soit la tendance à privilégier les souvenirs (ici, les anecdotes) les plus mémorables, c'est-à-dire ceux qui sont les plus vifs, les plus hors du commun, ou encore qui possèdent la plus grande charge émotive (Tversky et Kahneman, 1973), ce qui est cohérent avec l'effet de retour de flamme évoqué par Newton et Miah (2017). Pasquinelli (2012) a également évoqué l'heuristique de familiarité, soit la tendance à juger plus crédibles les souvenirs les plus familiers (c'est-à-dire, dans le cas qui nous préoccupe, ceux issus d'anecdotes personnelles), au détriment de d'autres (c'est-à-dire ceux issus de l'exposition au savoir scientifique). Enfin, Pasquinelli (2012) a évoqué le phénomène de l'amnésie de source (angl. source amnesia), qui consiste à oublier si l'origine d'un souvenir est fiable ou non (Schacter, Harbluk et McLachlan, 1984). La chercheure suggère que de tels biais cognitifs favorisent la persistance des neuromythes et souligne le besoin de mener des recherches pour découvrir quels sont les biais cognitifs qui favorisent le plus cette persistance.

\section{La présente étude}

Selon Grospietsch et Mayer (2018), les fausses croyances sur le cerveau et l'apprentissage étant profondément ancrées dans l'expérience personnelle, les interventions visant à démystifier les neuromythes devraient chercher à atteindre ce niveau de profondeur. Rappelons qu'une proportion élevée d'enseignants adhérant aux neuromythes, dans l'étude de Blanchette Sarrasin et al. (2019), ont évoqué des intuitions et des observations anecdotiques en classe pour justifier leur niveau d'accord. Ces données suggèrent que les anecdotes personnelles alimentent l'intensité d'une fausse croyance, vraisemblablement via le mécanisme du biais de confirmation (Riener et Willingham, 2010). Par ailleurs, lorsque leurs fausses croyances ont été confrontées au savoir scientifique, près du tiers des répondants de Newton et Miah (2017) ont indiqué vouloir malgré tout continuer d'en tenir compte dans

favorise l'apprentissage (Cancela et al., 2015; Hyatt, 2007; Spaulding, Mostert et Beam, 2010). C'est la raison pour laquelle plusieurs enquêtes internationales sur les neuromythes incluent, dans leur sondage, des énoncés tels que «De courtes périodes d'exercices de coordination peuvent améliorer l'intégration des hémisphères cérébraux gauche et droit » et «Des exercices répétitifs de coordination perceptivo-motrice peuvent améliorer les habiletés en lecture » (Dekker et al., 2012; traductions libres). 
leur pratique enseignante, $89 \%$ d'entre eux justifiant cette intention en évoquant leurs observations anecdotiques en classe. Ce résultat suggère que les anecdotes personnelles contribuent à protéger les neuromythes lorsque ceux-ci sont confrontés au savoir scientifique, vraisemblablement via les mécanismes de l'heuristique de disponibilité, de l'heuristique de familiarité et de l'amnésie de source (Pasquinelli, 2012).

Si les anecdotes personnelles vécues par les enseignants en classe alimentent l'intensité de leurs fausses croyances sur le cerveau et l'apprentissage, comme semblent le suggérer les données de Blanchette Sarrasin et al. (2019), et contribuent à protéger leurs fausses croyances de la confrontation avec le savoir scientifique, comme semblent le suggérer les données de Newton et Miah (2017), alors il semble raisonnable de spéculer que la création d'une nouvelle anecdote personnelle qui contredit une fausse croyance puisse servir d'élément perturbateur. C'est du moins la prémisse de la présente étude. Notre hypothèse stipulait qu'après avoir personnellement expérimenté l'inutilité pédagogique des styles d'apprentissage VAK dans le contexte d'une activité didactique, cette nouvelle anecdote personnelle serait susceptible de former, avec le savoir scientifique, une alliance suffisamment puissante pour faire contrepoids aux anecdotes déjà vécues par les répondants et déstabiliser la fausse croyance envers l'efficacité pédagogique des styles d'apprentissage VAK.

\section{Méthodologie}

\subsection{Participants}

Quarante et un étudiants francophones inscrits au programme de formation initiale à l'enseignement à l'École des sciences de l'éducation de l'Université Laurentienne (Sudbury, Ontario) ont participé à l'étude. Les participants ont été recrutés dans les cours offerts dans le cadre de leur programme de formation. En contrepartie de leur participation volontaire, les participants ont reçu des points bonis dans ces cours. Le seul critère d'inclusion était le bilinguisme, puisque deux questionnaires étaient en langue anglaise. L'échantillon était composé de 32 femmes et de 9 hommes, dont 36 âgés entre 21 et 30 ans, 3 entre 31 et 40 ans et 2 de plus de 40 ans. L'étude s'est déroulée de janvier à mars 2019, lors du second trimestre de la première année de formation des apprentis enseignants. Onze participants étaient inscrits à l'option 1 (cycles primaire et moyen : maternelle à $6^{\mathrm{e}}$ année), 13 à l'option 2 (cycles moyen et intermédiaire : $4^{\mathrm{e}}$ à $10^{\mathrm{e}}$ année) et 17 à l'option 3 (cycles intermédiaire et supérieur : $7^{\mathrm{e}}$ à $12^{\mathrm{e}}$ année).

\subsection{Chronologie de l'intervention}

Le protocole expérimental a reçu l'aval d'un comité institutionnel de déontologie de la recherche chez des êtres humains. En janvier 2019, après avoir été informés de la nature de leur participation et avoir signé un formulaire de consentement libre et éclairé, les participants ont rempli un sondage pré-expérimental sur leurs croyances envers les styles d'apprentissage VAK et sur leur intention de les utiliser dans leur pratique enseignante. Deux semaines plus tard, les répondants ont participé à une activité didactique possédant plusieurs ressemblances méthodologiques avec les mises à l'épreuve empiriques de l'hypothèse d'appariement publiées dans la littérature scientifique. Après l'activité, les participants ont été invités à remplir deux questionnaires mesurant le style d'apprentissage VAK prédominant, ainsi qu'une mesure d'auto-déclaration. Six semaines plus tard, en mars, les participants ont été conviés à une séance d'information au cours de laquelle nous leur avons dévoilé que leurs propres données personnelles (issues de l'activité didactique), tout comme la totalité des recherches scientifiques, disqualifiaient l'hypothèse d'appariement. Au terme de la séance d'information, les participants ont rempli un sondage post-expérimental sur leur intention d'utiliser les styles d'apprentissage VAK dans leur pratique enseignante.

\subsection{Sondage pré-expérimental}

Le sondage pré-expérimental contenait quatre énoncés sur le neuromythe des styles d'apprentissage VAK. Ces énoncés, étroitement inspirés de ceux de Tardif, Doudin et Meylan (2015), se limitaient à la distinction visuel/auditif. En effet, si plusieurs enseignants peuvent donner des exemples de pratiques pédagogiques destinées spécifiquement aux élèves 《 visuels » et « auditifs » (p. ex. utiliser un support visuel ou audio), peu d'entre eux peuvent donner des exemples de pratiques destinées spécifiquement aux élèves « kinesthésiques » (Ruhaak et Cook, 2018).

Les participants indiquaient leur niveau d'accord avec chaque énoncé sur une échelle de type Likert à quatre niveaux (1 = pas du tout d'accord; 2 = plutôt en désaccord; 3 = plutôt d'accord; 4 =tout à fait d'accord). Le premier énoncé, «Certaines personnes sont visuelles, d'autres sont auditives », visait à sonder l'adhésion à l'idée fausse de l'existence de catégories de personnes définies par la prédominance d'une modalité sensorielle. Le second énoncé, «Des études sur le cerveau ont démontré qu'il existe une distinction entre des personnes visuelles et auditives », visait à sonder la croyance en l'existence de fondements scientifiques à l'idée fausse de profils particuliers de fonctionnement cérébral basés sur une modalité sensorielle donnée. Le troisième énoncé, «Une approche pédagogique basée sur la distinction entre les élèves visuels et auditifs favorise l'apprentissage », visait à sonder directement l'adhésion à l'hypothèse d'appariement, sous-jacente au neuromythe des styles d'apprentissage VAK. Contrairement aux trois premiers énoncés, qui sondaient les croyances, le quatrième énoncé était de nature pratique : «Je prévois tenir compte de la distinction entre les élèves visuels et auditifs dans ma pratique d'enseignant(e) ».

\subsection{Activité didactique}

L'étude de Cuevas et Dawson (2018), décrite plus haut, a servi de source scientifique comparative à l'exercice didactique lors de la séance d'information. L'exercice didactique ne se voulait pas une reproduction intégrale du protocole expérimental utilisé par Cuevas et Dawson (2018). Cependant, 
notre activité didactique présentait plusieurs similitudes avec ce protocole. D'abord, tout comme ces auteurs, nous avons utilisé une tâche de mémorisation de mots dans laquelle les stimuli recevaient un traitement soit visuel, soit auditif. Ensuite, nous avons également utilisé une tâche de rappel indicé. Enfin, le questionnaire utilisé par Cuevas et Dawson (2018) pour déterminer le style VAK prédominant de leurs participants a également été utilisé dans la présente étude.

\subsubsection{Stimuli}

Une liste de 96 mots a été confectionnée. Les mots représentaient des objets concrets (p. ex. chat, klaxon, train) et contenaient d'une à quatre syllabes. Les mots ont été sélectionnés et couplés au hasard, de sorte à obtenir 48 paires de mots. La moitié de ces paires de mots (24 paires) ont été sélectionnées au hasard pour servir de stimuli dans la condition visuelle, tandis que les 24 autres paires de mots ont servi de stimuli dans la condition auditive. Quelle que soit la condition, les deux mots d'une paire étaient présentés côte à côte, séparés par un tiret, au centre d'une diapositive du logiciel Microsoft PowerPoint ${ }^{\circledR}$, en lettres minuscules, dans la police de caractères Calibri avec une taille de caractères de 44 points.

Nous avons tenu à utiliser des mots accompagnés d'images ou de sons, plutôt qu'uniquement des images et des sons, afin que les stimuli possèdent une certaine validité écologique. En effet, en contexte scolaire, on retrouve rarement des images ou des sons seuls, mais bien aussi des mots à l'écrit. Dans la condition visuelle, de sorte à accentuer l'aspect visuel des stimuli, les lettres formant les mots étaient visuellement rehaussées, à l'aide de coloris divers avec contraste figurefond. De plus, une image représentant chaque mot était présentée juste sous le mot. Par exemple, pour la paire de mots parapluie - cadeau, l'image d'un parapluie apparaissait sous le mot parapluie, tandis qu'une image de cadeau apparaissait sous le mot cadeau. En retour, dans la condition auditive, de sorte à accentuer l'aspect auditif des stimuli, une voix féminine préenregistrée prononçait les deux mots de la paire, de gauche à droite. La prononciation était lente, de sorte à « découper » chaque syllabe. De plus, juste après la voix enregistrée, un enregistrement de sons typiquement associés aux deux mots de la paire se faisait entendre. Par exemple, pour la paire de mots chat - cloche, un miaulement de chat était suivi, une seconde plus tard, par le tintement d'une cloche.

\subsubsection{Déroulement}

L'activité didactique a été introduite comme une autre des activités didactiques réalisées par les étudiants dans le cadre de leur formation et qui visent à les préparer pour les stages d'enseignement pratique. Les participants ont été informés que l'objectif de cette activité didactique particulière était de tester si les mots sont mieux retenus s'ils sont accompagnés d'images ou s'ils sont accompagnés de sons. Aucune mention des styles d'apprentissage « visuel » et « auditif » n'a été faite.

L'activité didactique s'est déroulée dans une salle équipée de postes d'ordinateurs. Comme la salle ne contenait que 32 postes, deux groupes aléatoires d'environ 20 participants ont réalisé l'activité, dans des conditions de passation identiques. Les participants portaient des écouteurs préréglés à un niveau sonore confortable. Les directives, transmises par écrit, étaient les suivantes :

Vous allez voir 48 paires de mots, une paire à la fois. Vingt-quatre paires de mots sont accompagnées d'images et 24 paires de mots sont accompagnées de sons. Votre tâche consiste à essayer de retenir le deuxième mot de chaque paire. Attention! Portez aussi attention au premier mot, car il servira plus tard d'indice pour vous souvenir du deuxième mot. Bonne chance!

La moitié des participants ont d'abord visionné les 24 diapositives de la condition visuelle, suivies des 24 diapositives de la condition auditive. L'ordre des deux conditions était inversé pour l'autre moitié des participants. Dans chaque condition, l'ordre de présentation des 24 diapositives était aléatoire. Le temps d'exposition de chaque diapositive était de 15 secondes, sans possibilité de retour en arrière, ni d'accélération de la vitesse de défilement. Dans la condition visuelle, les deux images étaient présentées pendant toute la durée d'exposition. Dans la condition auditive, les deux enregistrements (voix et sons) étaient présentés en succession, sous les 15 secondes d'exposition et sans répétition. La phase de mémorisation était d'une durée approximative de 12 minutes. Après le visionnement de la présentation Microsoft PowerPoint ${ }^{\circledR}$, les participants ont été invités à compléter un casse-tête de nombres combinatoires (Sudoku), pendant 15 minutes. Ce jeu servait de tâche distractrice.

Immédiatement après la tâche distractrice, les participants ont reçu un cahier contenant 48 items de rappel indicé, sous la forme mot - __ . Les 48 indices correspondaient aux 48 premiers mots des paires, présentés dans un ordre aléatoire d'un participant à un autre. Les directives spécifiaient qu'ils devaient tenter de se rappeler du deuxième mot de chaque paire, le premier mot servant d'indice. La consigne additionnelle «Fiez-vous à votre première impression » a été donnée aux participants, afin d'éviter que ceux-ci n'adoptent un critère de rappel trop conservateur, ce qui aurait pu donner lieu à un « effet plancher ». Un maximum de 15 minutes était dévolu au test de rappel indicé. Les participants avaient le loisir de revenir en arrière, advenant que le délai ne soit pas encore écoulé.

\subsection{Outils de mesure des styles d'apprentissage VAK}

Rappelons que le style d'apprentissage VAK prédominant des participants a été mesuré après l'activité didactique. L'ordre de passation des mesures des styles d'apprentissage VAK a été contrebalancé.

\subsubsection{Questionnaire VARK}

Le Visual Aural Read/Write Kinesthetic (VARK) Questionnaire (Fleming et Mills, 1992) est un instrument composé de 16 
items à choix multiples. Le répondant peut cocher plus d'une réponse. Voici un exemple d'item : «Vous êtes sur le point de donner des directives à une passante. Elle réside à l'hôtel et désire se rendre à votre maison. Elle a loué une auto. a) Je lui dessinerais une carte; b) Je lui donnerais des directives verbales; c) Je lui écrirais les directives sur un papier; (d) Je passerais la prendre à son hôtel avec ma voiture » (traduction libre). Leite, Svinicki et Shi (2010) ont réalisé une analyse factorielle confirmatoire et conclu que la structure du questionnaire VARK était conforme à l'existence de quatre facteurs sous-jacents, auxquels ces auteurs ont référé en tant que «traits latents » étiquetés visuel, oral, lecture/écriture (angl. read/write) et kinesthésique. Cependant, Leite et al. (2010) ont précisé que cette conclusion devait être considérée comme préliminaire, étant donné que les poids factoriels des variables étaient faibles à modérés. Leite et al. (2010) ont par ailleurs rapporté des coefficients de consistance interne adéquats, soit de $0,85,0,82,0,84$ et 0,77 pour les sous-échelles $\mathrm{V}, \mathrm{A}$, R et $\mathrm{K}$, respectivement.

\subsubsection{Inventaire BLSI}

Le Barsch Learning Style Inventory (BLSI; Barsch, 1991) est un instrument composé de 24 énoncés, suivis d'un choix de réponses (souvent vrai, parfois vrai, rarement vrai). Huit énoncés mesurent le style d'apprentissage visuel (p. ex. «J'ai besoin d'explications par des graphiques, des diagrammes et des directives visuelles »), huit énoncés le style auditif (p. ex. 《Je peux retenir mieux un sujet en écoutant qu'en lisant ») et huit énoncés le style tactile/kinesthésique (p. ex. «J'apprécie travailler avec des outils ou sur des modèles »; traductions libres). Aucune étude des qualités psychométriques de l'inventaire BLSI n'a été publiée. Cependant, Krätzig et Arbuthnott (2006) ont rapporté, chez leur échantillon $(n=65)$, des coefficients alpha de Cronbach en-deçà du seuil d'adéquation de 0,70 (Field, 2013) pour les sous-échelles visuelle $(0,54)$, auditive $(0,56)$ et tactile/kinesthésique $(0,38)$ du BLSI.

\subsubsection{Auto-déclaration}

Le style d'apprentissage VAK auto-déclaré a été obtenu à l'aide de la question suivante : «Je me considère comme une personne : a) plutôt visuelle qu'auditive; b) plutôt auditive que visuelle; c) à la fois visuelle et auditive; d) ni visuelle ni auditive ». À noter que le style kinesthésique n'était pas une option, en raison de l'absence de stimuli de nature kinesthésique dans l'activité didactique.

\subsubsection{Validité psychométrique des instruments}

Les deux instruments de mesure des styles VAK utilisés dans la présente étude (questionnaire VARK et inventaire BLSI) sont parmi les plus utilisés dans la littérature scientifique visant à tester l'hypothèse d'appariement (voir Rousseau et al., 2018). La très grande majorité des auteurs de ces tests empiriques reconnaissent d'emblée et soulignent à grands traits l'absence de validité psychométrique de ces instruments, mais les utilisent malgré tout pour classifier leurs participants, car ce sont des instruments auxquels réfèrent les tenants de «l'approche VAK 》 en pédagogie (p. ex. Othman et Amiruddin, 2010). D'ailleurs, selon certains auteurs (p. ex. Kozhevnikov, Evans et Kosslyn, 2014), l'invalidité des instruments de mesure des styles VAK constitue une limite méthodologique importante dans les mises à l'épreuve empiriques de l'hypothèse d'appariement.

Toutefois, dans le cadre de la présente étude, l'utilisation d'instruments invalides ne constitue pas une limite méthodologique, mais plutôt un avantage. D'une part, le choix de ces instruments permet de comparer directement les résultats de l'activité didactique aux résultats publiés dans la littérature scientifique sur l'hypothèse d'appariement. En effet, le questionnaire VARK a aussi été utilisé dans l'étude de Cuevas et Dawson (2018) ayant servi de source scientifique comparative à l'activité didactique. D'autre part, en raison de leur manque de validité, il était tout à fait prévisible que les scores obtenus aux deux instruments ne convergent pas. Or, cette absence de convergence a servi d'argument, au cours de la séance d'information, pour convaincre les participants que ces instruments (dont plusieurs versions informelles circulent sur internet et sont donc facilement accessibles aux enseignants) n'avaient aucune fiabilité. Cependant, les participants pouvaient être amenés à penser que leur propre évaluation personnelle de leur style d'apprentissage VAK serait plus fiable. C'est la raison pour laquelle nous avons également inclus une mesure d'auto-déclaration.

\subsection{Séance d'information}

La séance d'information à laquelle ont été conviés les apprentis enseignants comportait deux volets. Au cours du premier volet, le concept de style d'apprentissage VAK a été présenté :

Le «style d'apprentissage 》 VAK est une manière personnelle d'apprendre avec nos sens: certains élèves seraient davantage "visuels» (V), d'autres 《 auditifs » (A), et d'autres « kinesthésiques 》 (K).

Puis, l'inconsistance entre le style VAK déclaré par les élèves et le style VAK identifié par les enseignants, en milieu scolaire, a été soulignée, à l'aide de l'étude récente de PapadatouPastou, Gritzali et Barrable (2018) :

Dans une recherche de 2018, seulement $30 \%$ des élèves $d u$ primaire qui s'identifiaient comme 《visuels » ont été identifiés comme «visuels 》 par leur propre enseignant; pour les élèves «auditifs 》, c'était seulement $38 \%$ et pour les élèves «kinesthésiques 》, c'était seulement $52 \%$.

L'inconsistance entre les instruments de mesure des styles d'apprentissage VAK a également été soulignée aux apprentis enseignants, à l'aide de leurs propres résultats :

Les questionnaires officiels pour mesurer le style d'apprentissage VAK, auxquels vous avez répondu au laboratoire didactique, le VARK et le BLSI, ne sont pas plus fiables. Par exemple, 75 \% d'entre vous qui avez été identifiés comme «auditifs 》 par le 
VARK ont été identifiés comme «visuels» par le BLSI. Un autre exemple: $73 \%$ de ceux qui ont été identifiés comme «kinesthésiques» par le VARK ont été identifiés comme «visuels » par le BLSI. En plus, l'inventaire BLSI a réussi à identifier le style d'apprentissage que vous avez coché vous-même dans la question personnelle dans seulement $32 \%$ des cas. Pour le VARK, c'était seulement $37 \%$.

Le second volet de la séance d'information était consacré à l'hypothèse d'appariement. Le mot anglais matching a été utilisé, pour éviter toute incompréhension du terme appariement chez cet échantillon d'étudiants francoontariens. Le devis expérimental préconisé par Pashler et al. (2008) a servi de canevas :

Pour tester scientifiquement l'hypothèse de 'matching', il faut séparer les élèves en deux, par exemple ceux qui sont «visuels» d'après un questionnaire et ceux qui sont «auditifs ». Après, on présente la moitié du contenu pédagogique avec du support visuel, et l'autre moitié avec du support audio. Si l'hypothèse de 'matching' est valide, les élèves «visuels» devraient mieux apprendre le contenu avec du support visuel qu'avec du support audio. Pour les élèves auditifs, cela devrait être le contraire: ils devraient mieux apprendre le contenu avec du support audio qu'avec du support visuel [la figure 1 a été présentée pour illustrer cette interaction croisée STYLE $\times$ SUPPORT]. Si le groupe des «visuels》 et le groupe des «auditifs » apprennent mieux tous les deux le contenu avec le support visuel qu'avec le support audio, cela n'appuie pas l'hypothèse de 'matching'; cela veut simplement dire que le support visuel est plus efficace que le support audio, indépendamment $d u$ style d'apprentissage [la figure 2 a été présentée pour illustrer cet effet principal du SUPPORT].

Puis, les propres résultats des apprentis enseignants, issus de l'activité didactique, ont été présentés, à l'aide des figures 3 , 4 et 5 .

Vous voyez, peu importe la façon dont votre «style d'apprentissage» a été mesuré - par le questionnaire VARK, par l'inventaire BLSI ou par la question personnelle - les mots avec images ont toujours été mieux retenus que les mots avec sons, autant pour les «visuels » que pour les « auditifs ». Et même si vous avez coché que vous êtes une personne «à la fois visuelle et auditive», votre mémoire des mots avec images n'est pas égale à votre mémoire des mots avec sons : vous aussi avez mieux retenu les mots avec images.

\footnotetext{
${ }^{8}$ Cette figure a été présentée aux apprentis enseignants lors de la séance d'information (voir texte).
}

Les résultats de l'étude expérimentale de Cuevas et Dawson (2018) ont alors été présentés, à l'aide de la figure 2 de leur article (non reproduite ici), présentée côte à côte avec la figure 3 :

Dans une vraie recherche scientifique de 2018, les chercheurs ont obtenu des résultats très semblables à vos résultats personnels dans l'activité didactique : une meilleure mémoire pour les mots avec images que pour les mots avec sons. Eux aussi ont mesuré les styles d'apprentissage chez des étudiants universitaires avec le questionnaire VARK. Et eux non plus n'ont pas observé d'effet de 'matching'.

Dans les faits, Cuevas et Dawson (2018) n'ont pas présenté de mots accompagnés d'images et de sons. Leurs participants devaient soit imaginer visuellement laction exprimée par un énoncé, soit prononcer silencieusement l'énoncé. Cependant, tout comme dans notre activité didactique, les participants de Cuevas et Dawson(2018) devaient effectuer un traitement soit visuel, soit auditif de mots.

Enfin, une liste de références d'articles publiés dans des revues scientifiques arbitrées a été présentée :

En fait, il n'existe aucune recherche scientifique qui prouve que l'apprentissage est meilleur si on 'match' l'enseignement aux styles d'apprentissage VAK. La recherche montre plutôt que tel contenu pédagogique est mieux appris dans telle modalité sensorielle par la majorité des élèves, alors que tel autre contenu est mieux appris dans une autre modalité par la majorité des élèves.

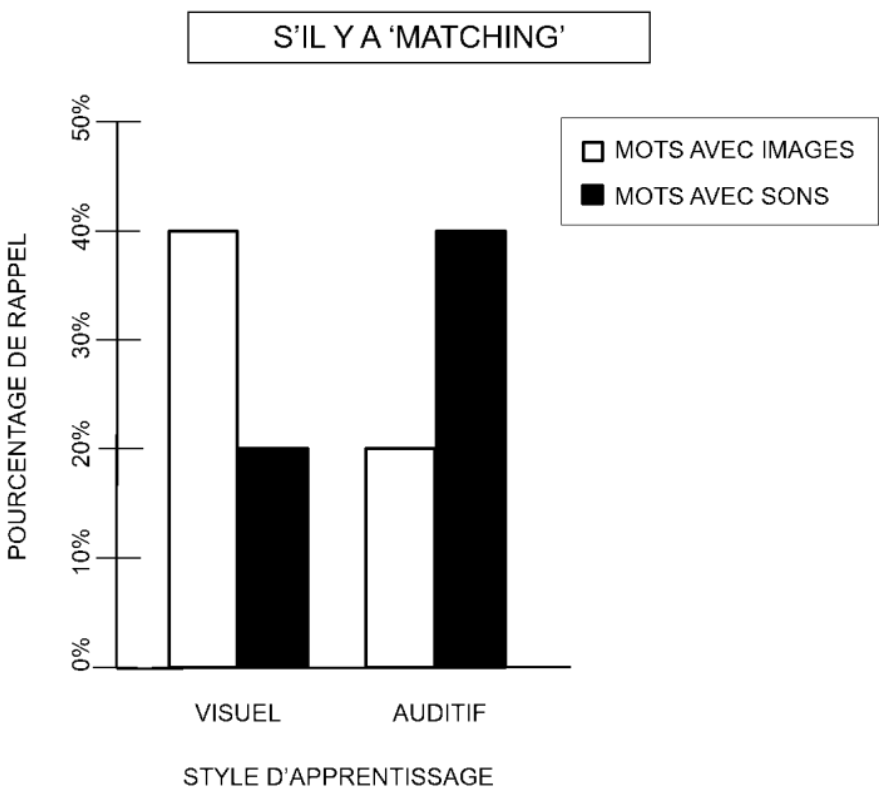

Figure 1. Profil de résultats conforme à l'hypothèse d'appariement (angl. matching hypothesis) entre les styles d'apprentissage VAK et la modalité sensorielle de présentation du contenu pédagogique. ${ }^{8}$ 


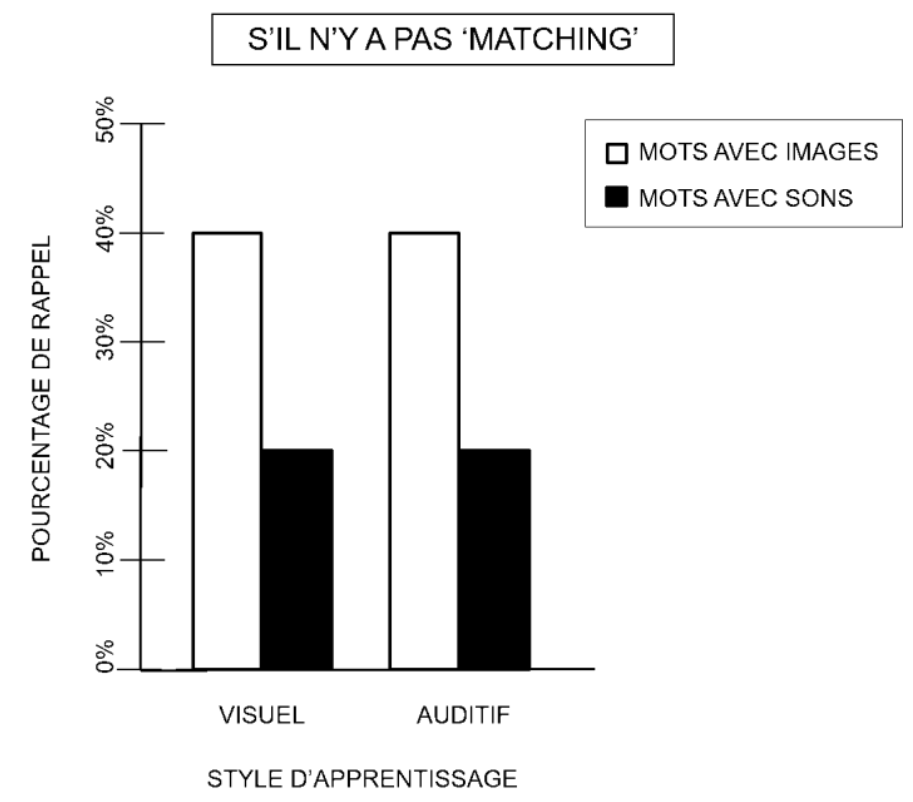

Figure 2. Profil de résultats non conforme à l'hypothèse d'appariement (angl. matching hypothesis) entre les styles d'apprentissage VAK et la modalité sensorielle de présentation du contenu pédagogique. ${ }^{9}$

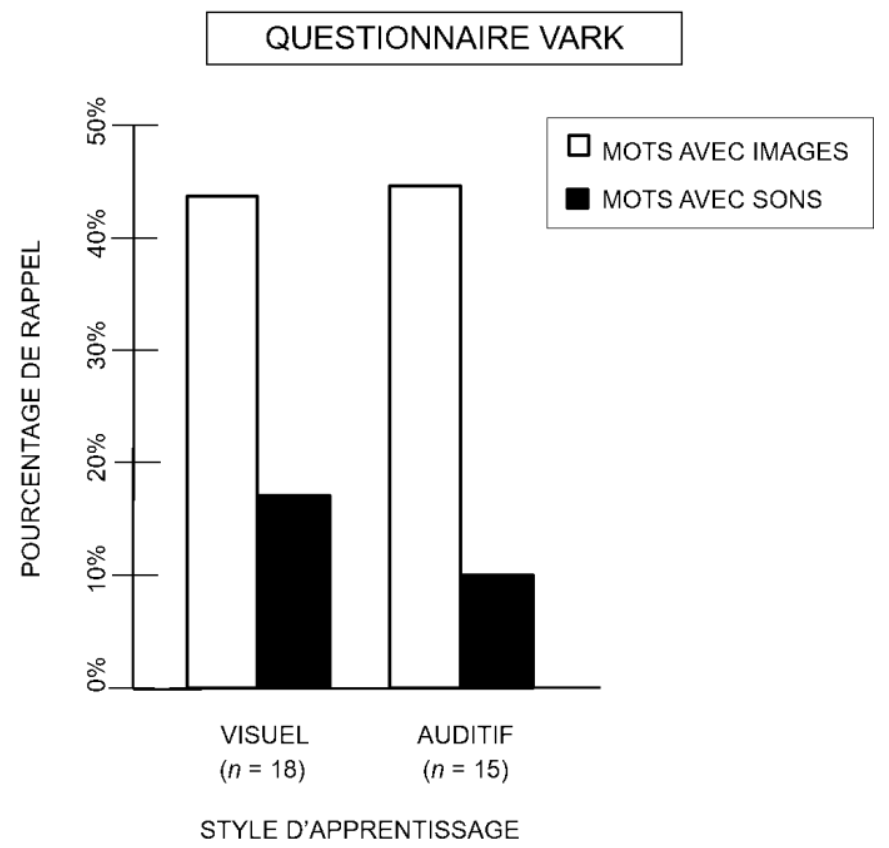

Figure 3. Profil de résultats issu de l'activité didactique, selon le questionnaire VARK. ${ }^{10}$

\footnotetext{
${ }^{9}$ Cette figure a été présentée aux apprentis enseignants lors de la séance d'information (voir texte).

${ }^{10}$ Lors de la séance d'information, cette figure a été présentée côte à côte avec la figure 2 (non reproduite ici) de l'article de Cuevas et Dawson (2018).
}

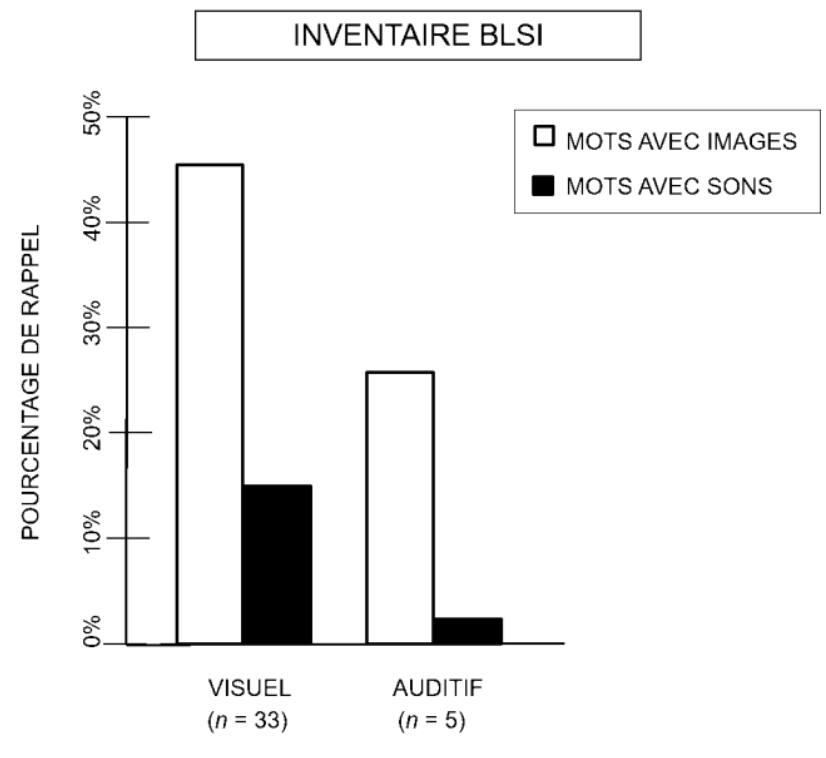

Figure 4. Profil de résultats issu de l'activité didactique, selon l'inventaire BLSI.

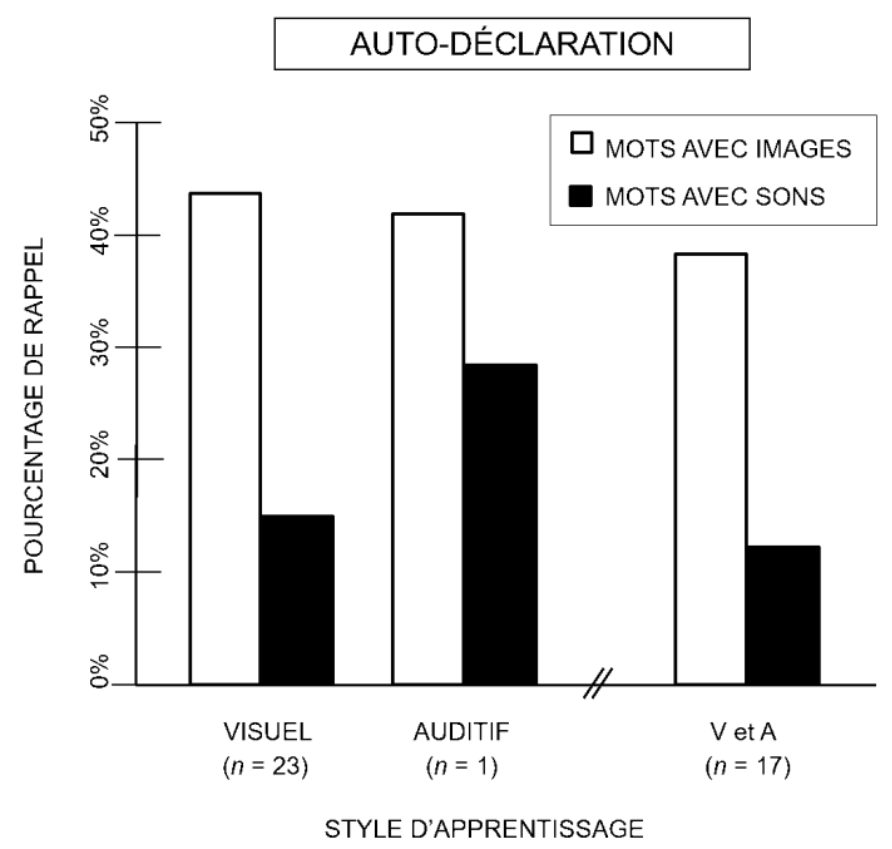

Figure 5. Profil de résultats issu de l'activité didactique, selon le style VAK auto-déclaré. ${ }^{12}$

${ }^{11}$ Cette figure a été présentée lors de la séance d'information (voir texte).

${ }^{12}$ «V et A » signifie personne à la fois visuelle et auditive. Cette figure a été présentée lors de la séance d'information (voir texte). 
En effet, tel que suggéré par Kozhevnikov et al. (2014), certains contenus pédagogiques sont mieux adaptés à certaines modalités sensorielles qu'à d'autres. Ainsi, enseigner le tango au téléphone serait vraisemblablement moins efficace que de l'enseigner à l'aide de mouvements (modalité kinesthésique). Quand il s'agit de compréhension de textes, une présentation orale (une bande sonore) s'avère meilleure qu'une présentation visuelle (un texte écrit), peu importe le style d'apprentissage prédominant des participants (Rogowsky, Calhoun et Tallal, 2015). Par ailleurs, quand il s'agit d'anatomie, c'est la modalité visuelle (l'utilisation d'un microscope virtuel) qui produit la meilleure note finale dans un cours, peu importe le style VAK prédominant des étudiants (Husmann et O'Loughlin, 2019). En somme, dans plusieurs études scientifiques, les chercheurs ont obtenu un effet principal de l'une ou l'autre modalité sensorielle de présentation du contenu pédagogique, un patron de résultats illustré par la figure 2 et qui correspond à la fois aux résultats obtenus par les participants lors de l'activité didactique (figures 3, 4 et 5) et aux résultats obtenus dans l'étude scientifique comparative de Cuevas et Dawson (2018). C'est la raison pour laquelle nous avons insisté, auprès des répondants, sur une modalité sensorielle à privilégier pour l'ensemble des élèves d'une classe, selon la nature du contenu pédagogique ${ }^{13}$.

\subsection{Sondage post-expérimental}

Le sondage post-expérimental était composé d'un seul énoncé, inspiré de celui de Newton et Miah (2017) :

Après avoir appris qu'il n'existe aucune preuve scientifique pour appuyer l'hypothèse selon laquelle 'matcher' l'enseignement aux styles d'apprentissage VAK (par exemple, présenter des images et des diagrammes aux apprenants visuels) améliore le rendement scolaire, je prévois tenir compte des styles d'apprentissage VAK dans ma pratique d'enseignant(e).

Les participants ont indiqué leur niveau d'accord sur une échelle de type Likert à quatre niveaux $(1=$ pas $d u$ tout d'accord; 2 = plutôt en désaccord; 3 = plutôt d'accord; 4 = tout à fait d'accord).

Nous avons fait le choix méthodologique de ne pas sonder à nouveau les répondants, à la suite de la séance d'information, sur les trois énoncés de croyance envers les styles d'apprentissage VAK utilisés lors de la phase préexpérimentale. Ce faisant, nous voulions éviter qu'un possible effet de désirabilité sociale, soupçonné par Newton et Miah (2017) en ce qui concerne les énoncés de croyance, ne « contamine » l'énoncé d'intention pratique.

\footnotetext{
${ }^{13}$ La recherche montre aussi que la présentation bimodale (p. ex., auditive et visuelle) du contenu pédagogique mène à un meilleur apprentissage (Shams et Seitz, 2008). Cependant, tenter de convaincre les enseignants
} que la présentation multisensorielle du contenu pédagogique est efficace

\section{Résultats}

\subsection{Sondage pré-expérimental}

Le tableau 2 présente les résultats du sondage préexpérimental. Comme c'est le cas dans plusieurs sondages, la prévalence d'adhésion aux énoncés correspondait au pourcentage de répondants ayant indiqué être plutôt d'accord ou tout à fait d'accord avec l'énoncé (c'est-à-dire les cotes 3 et 4 combinées sur l'échelle de type Likert à quatre niveaux). La prévalence d'adhésion à l'énoncé «Certaines personnes sont visuelles, d'autres sont auditives » était de $98 \%$, ce qui dénote une croyance presque unanime, chez l'échantillon, envers l'idée fausse de l'existence de catégories de personnes définies par une modalité sensorielle dominante. Le pourcentage d'apprentis enseignants adhérant à l'énoncé «Des études sur le cerveau ont démontré qu'il existe une distinction entre des personnes visuelles et auditives » était de $95 \%$. Cela dénote une forte croyance en l'existence de fondements scientifiques à l'idée fausse de profils particuliers de fonctionnement cérébral basés sur une modalité sensorielle donnée.

L'énoncé «Une approche pédagogique basée sur la distinction entre les élèves visuels et auditifs favorise l'apprentissage », qui contient l'hypothèse d'appariement, a recueilli l'adhésion de $93 \%$ des répondants. Il s'agit d'un énoncé de croyance. Mais tout comme l'ont observé Newton et Miah (2017) dans leur étude, le pendant pratique de cet énoncé a recueilli une adhésion encore plus forte : $100 \%$ des répondants ont indiqué être d'accord avec l'énoncé «Je prévois tenir compte de la distinction entre les élèves visuels et auditifs dans ma pratique d'enseignant(e) », dont $64 \%$ se disant tout à fait d'accord.

\subsection{Résultats aux mesures des styles d'apprentissage VAK}

En plus des styles d'apprentissage visuel et auditif, le questionnaire VARK mesure le style kinesthésique et le style «lecture/écriture » (angl. read/write), alors que l'inventaire BLSI mesure également le style kinesthésique. L'activité didactique ne comportant ni de condition kinesthésique ni de condition «lecture/écriture », la procédure de reclassement utilisée par Cuevas et Dawson (2018) a été appliquée, afin de déterminer lequel, parmi deux styles d'apprentissage - visuel ou auditif -, était prédominant chez les participants. Ainsi, lorsque le score de classification prédominant était kinesthésique ou "lecture/écriture », le second style prédominant (visuel ou auditif) a été retenu. De même, quand le score de classification prédominant au BLSI ou au VARK était bimodal, la modalité collatérale (visuelle ou auditive) a été retenue (p. ex. un répondant classifié comme kinesthésique-visuel au BLSI était reclassifié comme visuel).

pour une raison autre que l'existence de styles d'apprentissage VAK représente un défi considérable. Nous reviendrons sur ce point à la section 5.4. 
Au VARK, lorsque le score bimodal ne comportait ni visuel ni auditif (c'est-à-dire lecture/écriture-kinesthésique), le troisième style prédominant (visuel ou auditif) a été retenu. Enfin, lorsque le premier, second ou troisième score prédominant était bimodal (c'est-à-dire visuel-auditif), le répondant a été retiré de l'échantillon pour le calcul des résultats de l'activité didactique. Au total, $66 \%$ de scores au VARK (27 participants) ont été reclassés, tandis que le pourcentage de reclassement des scores au BLSI était de $29 \%$ (12 participants). Par ailleurs, huit participants (20\%) ont été retirés en raison d'un score bimodal visuel-auditif (original ou reclassé) au VARK, tandis que trois participants (7\%) ont été retirés pour cette même raison au BLSI.

Tableau 2. Répartition du niveau d'accord et prévalence pour quatre énoncés sur le neuromythe des styles d'apprentissage VAK.

\begin{tabular}{|c|c|c|c|c|c|}
\hline & \multicolumn{4}{|c|}{ Niveau d'accord* } & \multirow{2}{*}{$\begin{array}{c}\text { Prévalence } \\
3+4\end{array}$} \\
\hline & 1 & 2 & 3 & 4 & \\
\hline \multicolumn{6}{|l|}{ Énoncés de croyance } \\
\hline $\begin{array}{l}\text { Certaines personnes sont } \\
\text { visuelles, d'autres sont } \\
\text { auditives. }\end{array}$ & $0 \%$ & $2 \%$ & $44 \%$ & $54 \%$ & $98 \%$ \\
\hline $\begin{array}{l}\text { Des études sur le cerveau } \\
\text { ont démontré qu'il existe } \\
\text { une distinction entre des } \\
\text { personnes visuelles et } \\
\text { auditives. }\end{array}$ & $0 \%$ & $5 \%$ & $71 \%$ & $24 \%$ & $95 \%$ \\
\hline $\begin{array}{l}\text { Une approche pédagogique } \\
\text { basée sur la distinction } \\
\text { entre les élèves visuels et } \\
\text { auditifs favorise } \\
\text { l'apprentissage. }\end{array}$ & $2 \%$ & $5 \%$ & $39 \%$ & $54 \%$ & $93 \%$ \\
\hline \multicolumn{6}{|l|}{ Énoncé pratique } \\
\hline $\begin{array}{l}\text { Je prévois tenir compte de } \\
\text { la distinction entre les } \\
\text { élèves visuels et auditifs } \\
\text { dans ma pratique } \\
\text { d'enseignant(e). }\end{array}$ & $0 \%$ & $0 \%$ & $37 \%$ & $63 \%$ & $100 \%$ \\
\hline
\end{tabular}

* (1) pas du tout d'accord, (2) plutôt en désaccord, (3) plutôt d'accord, (4) tout à fait d'accord

Après reclassement et retraits, $55 \%$ des participants ont été classifiés comme « visuels », tandis que $45 \%$ ont été classifiés comme «auditifs » par le questionnaire VARK. En retour, $87 \%$ des participants ont été classifiés comme «visuels», tandis que $13 \%$ ont été classifiés comme «auditifs»par l'inventaire BLSI.

En ce qui concerne la mesure d'auto-déclaration, $56 \%$ des répondants ont déclaré se considérer comme une personne plutôt visuelle qu'auditive. Un seul répondant a déclaré se considérer comme une personne plutôt auditive que visuelle. En fait, deux apprentis enseignants sur cinq (41\%) ont déclaré se considérer comme une personne à la fois visuelle et auditive. À noter qu'aucun répondant n'a déclaré se considérer comme une personne ni visuelle ni auditive.

\subsection{Résultats de l'activité didactique}

Les figures 3 et 4 illustrent le score de rappel indicé, exprimé en pourcentage, pour les mots accompagnés d'images et les mots accompagnés de sons, en fonction du style d'apprentissage prédominant (visuel ou auditif), après reclassements et retraits, au VARK et au BLSI, respectivement. La figure 5 illustre, quant à elle, le score de rappel indicé en fonction du style auto-déclaré. Pour l'autodéclaration, en raison du grand nombre de répondants (41\%) ayant déclaré se considérer comme une personne à la fois visuelle et auditive, nous avons préservé cette catégorie à des fins démonstratives.

Les résultats de l'activité didactique sont clairs : quel que soit le style d'apprentissage VAK prédominant (visuel ou auditif) d'après le questionnaire VARK ou l'inventaire BLSI, le score de rappel indicé était nettement plus élevé pour les mots accompagnés d'images que pour les mots accompagnés de sons. Le même patron de résultats a été obtenu selon le style d'apprentissage auto-déclaré (y compris pour la catégorie une personne à la fois visuelle et auditive). Il est important de mentionner que l'activité didactique ne visait pas à tester de manière formelle l'hypothèse d'appariement. Il ne s'agissait pas d'une expérience scientifique, mais bien d'une activité didactique réalisée dans le seul but de créer une anecdote personnelle chez les apprentis enseignants. C'est pourquoi les données n'ont fait l'objet d'aucune analyse statistique. De telles analyses auraient d'ailleurs nécessité une taille d'échantillon beaucoup plus considérable. Puisque notre objectif consistait à présenter un patron de résultats conforme à l'absence d'effet d'appariement (voir figure 2), la seule illustration des moyennes était suffisante.

\subsection{Sondage post-expérimental}

Le tableau 3 présente la répartition du niveau d'accord avec l'unique énoncé du sondage post-expérimental :
Après avoir appris qu'il n'existe aucune preuve scientifique pour appuyer l'hypothèse selon laquelle 'matcher' l'enseignement aux styles d'apprentissage VAK (par exemple, présenter des images et des diagrammes aux apprenants visuels) améliore le rendement scolaire, je prévois tenir compte des styles d'apprentissage VAK dans ma pratique d'enseignant(e).

À la suite de notre intervention, le pourcentage d'apprentis enseignants ayant l'intention d'utiliser les styles d'apprentissage VAK dans leur pratique enseignante (cotes 3 et 4 combinées) a décliné, passant de $100 \%$ (taux préexpérimental) à $60 \%$, soit une baisse d'intention de $40 \%$. Soulignons que le pourcentage de répondants ayant coché plutôt en accord est resté inchangé (37\% au pré-test vs. $38 \%$ au post-test). Cependant, le nombre de répondants ayant coché tout à fait d'accord est passé de $63 \%$ à $22 \%$, une baisse 
de $41 \%$. Finalement, $16 \%$ des répondants ont totalement abandonné cette intention, cochant pas du tout d'accord.

Un test de Wilcoxon a révélé une différence significative entre la cote d'accord médiane pré-expérimentale (4.00) et la cote d'accord médiane post-expérimentale (3.00), $z=-4.03$, $p<0,001, r=0,44^{14}$.

Tableau 3. Répartition du niveau d'accord et prévalence pour l'énoncé pratique sur le neuromythe des styles d'apprentissage VAK, à la suite de la séance d'information.

\begin{tabular}{lccccc}
\hline & \multicolumn{3}{c}{ Niveau d'accord* } & Prévalence \\
\hline & 1 & 2 & 3 & 4 & $3+4$ \\
Énoncé pratique & & & & & \\
\hline $\begin{array}{l}\text { Après avoir appris qu'il } \\
\text { n'existe aucune preuve }\end{array}$ & $16 \%$ & $24 \%$ & $38 \%$ & $22 \%$ & $60 \%$ \\
scientifique pour appuyer & & & & & \\
l'hypothèse selon laquelle & & & & & \\
'matcher' l'enseignement & & & & & \\
aux styles d'apprentissage \\
VAK (par exemple, \\
présenter des images et \\
des diagrammes aux \\
apprenants visuels) \\
améliore le rendement \\
scolaire, je prévois tenir \\
compte des styles \\
d'apprentissage VAK dans \\
ma pratique \\
d'enseignant(e).
\end{tabular}

* (1) pas du tout d'accord, (2) plutôt en désaccord, (3) plutôt d'accord, (4) tout à fait d'accord

\section{Discussion}

\subsection{Sondage pré-expérimental}

À notre connaissance, aucune donnée n'est encore disponible sur la prévalence d'adhésion aux neuromythes (dans leur ensemble) en Ontario. Notre sondage indique un taux d'adhésion de $93 \%$ au neuromythe des styles d'apprentissage VAK chez un échantillon d'apprentis enseignants francoontariens en formation initiale $(n=41)$. Un tel taux se compare à la prévalence observée chez des échantillons représentatifs de la même population en Australie (97 \%; Kim et Sankey, 2018) et en Grèce (94 \%; Papadatou-Pastou et al., 2017) au cours des deux dernières années (la prévalence était de $82 \%$ au Royaume-Uni il y a dix ans; Howard-Jones et al., 2009).

Ailleurs au Canada, à notre connaissance, les seules autres données disponibles auprès d'apprentis enseignants sont celles (non publiées) de Loi Zedda (2014), obtenues auprès d'échantillons d'étudiants québécois en formation initiale à l'enseignement préscolaire et primaire $(n=59)$ ou spécialisée en adaptation scolaire et sociale $(n=23)$. L'énoncé du neuromythe des styles d'apprentissage VAK utilisé par Loi Zedda (2014) était semblable à celui utilisé dans la présente étude : «Adapter son enseignement en fonction du profil de l'élève (auditif, visuel et kinesthésique) favorise ses apprentissages ». Les répondants indiquaient leur degré d'accord avec l'énoncé sur une échelle de type Likert à cinq niveaux, allant de (1) pas du tout d'accord à (5) tout à fait d'accord (les niveaux 2, 3 et 4 n'étaient pas explicitement qualifiés). Plutôt que le pourcentage de participants ayant coché chacune des cinq catégories de réponses, Loi Zedda (2014) a rapporté la cote moyenne d'accord, sur cinq. La cote moyenne d'accord était de 4,5 et de 4,7/5 chez les apprentis enseignants québécois en formation initiale et spécialisée, respectivement. Bien que notre échelle de type Likert ne contienne que quatre niveaux, la cote moyenne d'accord chez notre échantillon d'apprentis enseignants franco-ontariens à l'énoncé «Une approche pédagogique basée sur la distinction entre les élèves visuels et auditifs favorise l'apprentissage » était de $3,4 / 4$, soit $4,3 / 5$, ce qui pourrait suggérer un taux d'adhésion au neuromythe des styles d'apprentissage VAK relativement comparable entre le Québec et l'Ontario chez les apprentis enseignants.

Cependant, l'enquête récente de Blanchette Sarrasin et al. (2019), réalisée auprès d'un vaste échantillon $(n=972)$ d'enseignants québécois francophones (aux niveaux préscolaire, primaire et secondaire), a révélé une prévalence moindre d'adhésion aux neuromythes. Dans cette enquête, la prévalence d'adhésion au neuromythe des styles d'apprentissage VAK était de $74 \%$. De l'avis même des auteurs, les taux de prévalence observés pour les cinq neuromythes sondés étaient plus bas que ceux rapportés dans des enquêtes précédentes ailleurs dans le monde, bien qu'ils demeurent élevés. Blanchette Sarrasin et al. (2019) ont évoqué la possibilité que cette prévalence moindre soit spécifique au Québec, ou encore, que la croyance internationale aux neuromythes soit en déclin. Puisque notre échantillon était constitué d'apprentis enseignants et non d'enseignants en exercice, il serait pertinent de réaliser une vaste enquête provinciale, en Ontario, auprès d'enseignants en exercice (en prenant en considération la langue d'enseignement), pour vérifier si la situation observée dans la profession enseignante au Québec se généralise ailleurs au Canada.

\subsection{Intervention}

L'originalité de notre étude reposait sur la création, chez les répondants, d'une anecdote personnelle dans laquelle l'hypothèse d'appariement était disqualifiée, comme c'est le cas dans la totalité des recherches scientifiques sur les styles d'apprentissage VAK (Rousseau et al., 2018). Notre hypothèse stipulait qu'en raison d'une telle convergence, l'expérience personnelle et le savoir scientifique formeraient une alliance suffisamment puissante pour faire contrepoids aux

\footnotetext{
${ }^{14}$ Une fois converti, lindice de puissance $r=0,44$ correspond à un $d$ de Cohen $=0,98$ (taille d'effet forte).
} 
anecdotes déjà vécues par les répondants et déstabiliser la fausse croyance envers l'utilité pédagogique des styles d'apprentissages VAK.

Dans la mesure où lintention de tenir compte des styles d'apprentissage VAK dans leur pratique enseignante reflète possiblement la croyance envers l'utilité pédagogique des styles VAK, la baisse statistiquement significative de ce taux d'intention, chez nos participants, appuie notre hypothèse. Cependant, le taux considérable de résistance à l'intervention (60 \%) laisse suggérer que l'anecdote créée n'avait pas une puissance suffisante pour faire contrepoids aux autres anecdotes personnelles vécues dans le passé des participants. Nous reviendrons sur ce point dans la section 5.4 .

Nous allons maintenant comparer notre intervention à celle réalisée par Newton et Miah (2017), bien que nos études respectives présentent un certain nombre de différences méthodologiques. L'intervention de Newton et Miah (2017) a été réalisée au Royaume-Uni, chez un échantillon composé de professeurs britanniques aux niveaux collégial et universitaire possédant, en moyenne, 11 ans d'expérience professionnelle en enseignement. Notre échantillon était plutôt composé d'apprentis enseignants canadiens inscrits en première année d'un programme de formation initiale à l'enseignement. En ce qui concerne la nature de l'intervention, celle de Newton et Miah (2017) reposait sur deux éléments: le manque de preuves scientifiques et la présentation d'énoncés sur les torts que peut causer l'utilisation du concept de styles d'apprentissage en classe. Notre propre intervention reposait, quant à elle, sur l'absence de preuves scientifiques et sur l'évocation d'une anecdote personnelle (issue d'une activité didactique) dans laquelle les répondants ont personnellement expérimenté l'absence de telles preuves.

En dépit de différences méthodologiques manifestes, notre intervention n'a pas été aussi efficace, en termes absolus, que celle de Newton et Miah (2017). Dans leur étude, de $64 \%$ avant l'intervention, le pourcentage de professeurs ayant l'intention d'utiliser le concept de styles d'apprentissage dans leur pratique enseignante a décliné à $32 \%$ après l'intervention, une chute de moitié. Dans la présente étude, de $100 \%$ avant l'intervention, le pourcentage d'apprentis enseignants ayant l'intention d'utiliser ce concept dans leur pratique enseignante a décliné à $60 \%$ après l'intervention, une chute qui n'atteint pas la moitié. Pourquoi un taux aussi considérable $(60 \%)$ de résistance à l'intervention?

À l'instar de Newton et Miah (2017), nous pourrions évoquer un effet de retour de flamme, possiblement plus accentué chez les apprentis enseignants en première année de formation que chez les professeurs d'expérience au collège ou à l'université. En effet, il se peut que les apprentis enseignants entretiennent des attentes plus idéalistes que les professeurs envers l'efficacité de pratiques pédagogiques inspirées des styles d'apprentissage, ce qui pourrait avoir provoqué une réaction antagoniste plus intense au savoir scientifique. L'effet de retour de flamme pourrait également avoir été plus accentué, chez notre échantillon, en raison de la nature plus personnelle de notre intervention. En effet, par la création d'une anecdote personnelle, nos répondants ont été confrontés de manière plus directe à leurs croyances profondes que ceux de Newton et Miah (2017), ce qui pourrait avoir engendré une réaction à charge émotive plus élevée. À l'appui de cette spéculation, à la fin de notre séance d'information, un apprenti enseignant, visiblement ébranlé sur le plan émotif, nous a confié qu'il vérifierait nos dires.

Outre un effet de retour de flamme possiblement plus accentué chez les apprentis enseignants que chez les professeurs, le taux considérable de résistance à l'intervention (60 \%) observé dans la présente étude pourrait relever de lacunes présentes dans le libellé de l'énoncé utilisé lors du sondage post-expérimental :
Après avoir appris qu'il n'existe aucune preuve scientifique pour appuyer l'hypothèse selon laquelle 'matcher' l'enseignement aux styles d'apprentissage VAK (par exemple, présenter des images et des diagrammes aux apprenants visuels) améliore le rendement scolaire, je prévois tenir compte des styles d'apprentissage VAK dans ma pratique d'enseignant(e).

En effet, ce libellé fait mention explicite de l'absence de preuves scientifiques à l'appui de l'utilité pédagogique des styles d'apprentissage VAK, mais n'évoque pas à nouveau l'anecdote personnelle issue de l'activité didactique. Même si cet énoncé a été présenté immédiatement après la présentation conjointe des résultats de l'étude scientifique de Cuevas et Dawson (2018) et des résultats de l'activité didactique, il demeure possible que les répondants n'aient pris en considération, pour évaluer leur intention personnelle d'utiliser les styles d'apprentissage VAK dans leur pratique enseignante, uniquement l'élément scientifique. Par conséquent, un énoncé évoquant à la fois le savoir scientifique et l'anecdote personnelle aurait possiblement pu donner lieu à un taux de résistance plus faible.

\subsection{Limites de la présente étude}

En dépit du caractère original et de l'efficacité potentielle de notre intervention, son application particulière dans la présente étude n'est pas sans présenter certaines limites. Premièrement, le profil de résultats obtenu lors de l'activité didactique (un score moyen de rappel indicé nettement supérieur pour les mots accompagnés d'images plutôt que de sons) pourrait avoir été biaisé par des attentes créées lors du sondage pré-expérimental sur les styles d'apprentissage VAK, administré deux semaines auparavant. En effet, il est possible que les participants aient mis plus d'effort à retenir les mots présentés dans la condition correspondant à leur style VAK préféré. Cependant, dans l'étude de Knoll, Otani, Skeel et Van Horn (2017), les participantes (uniquement des femmes) devaient mémoriser des paires de mots et des paires d'images. Pour chaque paire de mots ou d'images, elles devaient émettre un jugement subjectif sur leur capacité de se souvenir du deuxième item si le premier item était 
présenté comme indice. À la suite de la phase de mémorisation et immédiatement avant la phase de rappel indicé, Knoll et al. (2017) ont demandé aux participantes si elles croyaient que le rappel serait meilleur quand le format de présentation des items était apparié à leur style d'apprentissage. Toutes les participantes (100 \%) ont répondu par l'affirmative. Elles devaient aussi cocher si leur style d'apprentissage personnel était davantage visuel ou verbal. Or, en dépit de la création explicite d'attentes et de l'évocation de leur propre style d'apprentissage immédiatement avant la tâche de rappel indicé, les images ont été mieux rappelées par les participantes, indépendamment de leurs jugements subjectifs (prédictions de rappel) lors de la phase de mémorisation et indépendamment de leur style d'apprentissage (visuel ou verbal) auto-déclaré ou déterminé par leur score à la version révisée du Verbalizer-Visualizer Questionnaire (VVQ; Kirby, Moore et Schofield, 1988). Toutefois, il n'est pas exclu que des attentes aient pu être créées dans la présente étude. Â noter que la très grande majorité des études expérimentales publiées dans la littérature scientifique visant à tester l'hypothèse d'appariement souffrent potentiellement du même biais, les instruments de mesure des styles d'apprentissage VAK étant administrés en amont de la tâche expérimentale (c'est le cas de sept des huit études recensées par Rousseau et al., 2018). Dans la présente étude, les trois mesures (questionnaire VARK, inventaire BLSI et autodéclaration) ont, au contraire, été administrées en aval de la tâche expérimentale.

Deuxièmement, nous avons délibérément choisi de ne pas sonder à nouveau les répondants, après la séance d'information, sur les trois énoncés de croyance envers les styles d'apprentissage VAK utilisés lors de la phase préexpérimentale. Nous ignorons donc si la croyance en tant que telle envers ce neuromythe a diminué à la suite de notre intervention. Notre justification pour ce choix méthodologique est toutefois discutable. En effet, nous voulions éviter qu'un possible effet de désirabilité sociale, soupçonné par Newton et Miah (2017) en ce qui concerne les énoncés de croyance, ne «contamine » notre énoncé d'intention pratique. Or, pour éviter une telle contamination, nous aurions pu choisir de présenter, dans le sondage postexpérimental, l'énoncé d'intention pratique avant les énoncés de croyance. De plus, en dépit de l'absence d'énoncés de croyance au post-test, l'énoncé d'intention pratique a tout aussi bien pu, en soi, être victime d'un effet de désirabilité sociale, de sorte que le taux réel de résistance pourrait s'avérer plus élevé que le taux observé (60\%). Par conséquent, l'absence d'énoncés de croyance au sondage post-expérimental constitue une limite importante de la présente étude.

Troisièmement, la baisse significative de l'intention des apprentis enseignants d'utiliser les styles d'apprentissage VAK dans leur pratique enseignante, observée dans la présente étude, ne peut être formellement attribuée à l'évocation d'une anecdote personnelle disqualifiant leur utilité pédagogique. Toute autre composante de la séance d'information pourrait avoir causé cet effet. Seul un protocole expérimental rigoureux, comprenant trois échantillons d'apprentis enseignants issus de la même cohorte, serait susceptible de dissocier l'effet relatif de l'exposition au savoir scientifique et de l'évocation d'une anecdote personnelle : un groupe expérimental exposé à la fois au savoir scientifique et à une anecdote personnelle, vs. un groupe témoin exposé uniquement au savoir scientifique, vs. un groupe témoin exposé uniquement à une anecdote personnelle. L'absence de tels groupes témoins constitue une autre limite importante de la présente étude.

\subsection{Recherches futures}

Dans la présente recherche, nous avons misé sur une intervention axée sur une alliance entre le savoir scientifique et l'évocation d'une anecdote personnelle pour disqualifier l'efficacité pédagogique des styles d'apprentissage VAK. Or, il est possible que nous ayons sous-estimé la puissance des anecdotes personnelles déjà vécues par les participants, puisque l'alliance de notre nouvelle anecdote personnelle et du savoir scientifique n'a pas été suffisante pour susciter, chez $60 \%$ d'entre eux, une baisse de l'intention d'utiliser les styles d'apprentissage VAK dans leur pratique enseignante. Pourquoi un taux aussi considérable $(60 \%)$ de résistance à l'intervention?

Un manque de puissance de l'anecdote personnelle créée lors de l'activité didactique pourrait possiblement expliquer le taux élevé de résistance à l'intervention. Rappelons que deux apprentis enseignants sur cinq (41\%), dans notre échantillon, ont déclaré se considérer comme une personne à la fois visuelle et auditive. Pourtant, la presque totalité (98\%) des répondants ont indiqué être d'accord avec l'énoncé «Certaines personnes sont visuelles, d'autres sont auditives ». Il se peut que les répondants croient davantage en l'existence d'une modalité sensorielle dominante chez les enfants et moins chez les adultes. Si tel est le cas, de manière à accroître la puissance de notre anecdote, l'activité didactique pourrait être réalisée chez un groupe d'élèves d'une école primaire, plutôt que chez les apprentis enseignants eux-mêmes. Ceux-ci seraient ainsi directement témoins de l'inefficacité des styles d'apprentissage VAK à prédire une performance scolaire (ici, un test de rappel indicé) chez les élèves. De plus, une activité didactique réalisée en milieu éducatif serait susceptible de susciter, chez les répondants, une charge émotive plus élevée (rappelons que l'heuristique de disponibilité implique l'évocation d'un souvenir à forte charge émotive).

Cependant, nos répondants étaient des apprentis enseignants en première année de formation initiale à l'enseignement. Des enseignants en exercice depuis un certain nombre d'années disposent probablement de beaucoup plus d'anecdotes personnelles, vécues dans le passé, qui semblent confirmer l'efficacité pédagogique des styles d'apprentissage VAK. Considérant le taux de résistance déjà élevé observé chez des apprentis enseignants disposant de moins d'expérience d'enseignement, nous pouvons 
supposer un taux de résistance encore plus élevé chez des enseignants en exercice. Toutefois, il demeure possible qu'une activité didactique réalisée chez leurs propres élèves puisse s'avérer efficace. En effet, dans un tel protocole expérimental, les anciennes observations anecdotiques favorables au neuromythe des styles d'apprentissage VAK et la nouvelle anecdote défavorable créée lors de l'activité didactique seraient issues de leurs propres élèves, une situation qui pourrait possiblement favoriser, chez les enseignants en exercice, une déstabilisation plus marquée de leurs fausses croyances.

L'étude de Grospietsch et Mayer (2018) suggère une piste de recherche prometteuse sur les interventions à privilégier. Ces auteurs ont misé sur des textes de changement conceptuel pour tenter d'atteindre plus en profondeur les systèmes de croyance de leurs participants. Cependant, tel que mentionné précédemment, si cette intervention semble avoir diminué l'adhésion aux neuromythes, nous ignorons si ce résultat est victime d'un effet de désirabilité sociale, comme cela semble avoir été le cas dans l'étude de Newton et Miah (2017). Conséquemment, l'étude de Grospietsch et Mayer (2018) ne permet ni de conclure en une baisse réelle de croyance aux neuromythes, ni en une diminution de l'intention de tenir compte des neuromythes dans la pratique enseignante, puisque cette intention n'a pas été sondée dans cette recherche. Cependant, puisque l'intervention de Grospietsch et Mayer (2018) permettrait d'atteindre plus en profondeur l'expérience personnelle des participants (en raison des textes de changement conceptuel) que celle de Newton et Miah (2017), il est plausible qu'une telle intervention puisse également avoir un impact sur les intentions pratiques. Une étude reprenant l'intervention en deux composantes (exposition aux neurosciences cognitives de l'apprentissage et textes de changement conceptuel) de Grospietsch et Mayer (2018), avec non seulement une comparaison pré/post de l'adhésion aux neuromythes, mais également de l'intention d'en tenir compte dans la pratique enseignante, serait sans doute la bienvenue.

Si les pratiques pédagogiques inspirées des neuromythes sont si réfractaires aux interventions, comme le suggère l'étude de Newton et Miah (2017), alors il serait pertinent de s'interroger plus avant sur la nature de telles pratiques. À notre connaissance, aucune étude systématique n'a été réalisée, jusqu'à présent, pour connaître les pratiques pédagogiques mises en place par les enseignants pour «respecter» les styles d'apprentissage de leurs élèves. Cependant, nous disposons de quelques données qualitatives. Notamment, Sharp et al. (2008) ont rapporté les propos d'une enseignante du primaire, propos que ces auteurs considèrent représentatifs des réponses recueillies à l'aide de 76 questionnaires distribués au Royaume-Uni : «J'enseigne depuis bientôt 30 ans et j'ai toujours reconnu que les enfants apprennent de manières différentes. J'ai toujours essayé d'inclure un éventail d'expériences dans mon enseignement pour accommoder les différents styles d'apprentissage » (p. 302; traduction libre). De même, dans l'étude de Newton et Miah (2017), les professeurs britanniques aux niveaux collégial et universitaire pouvaient laisser un commentaire libre. Fait intéressant, le thème dominant de ces commentaires était le besoin d'utiliser une variété de méthodes d'enseignement pour garder actifs (angl. engaged) les étudiants et favoriser la réflexion. Parmi les 48 répondants ayant laissé des commentaires, 23 ont mentionné ce besoin de diversité, ajoutant, dans plusieurs cas, «en dépit de l'absence de preuves envers l'efficacité pédagogique des styles d'apprentissage » (traduction libre). D'ailleurs, Riener et Willingham (2010), qui ont pourtant vivement critiqué le concept de styles d'apprentissage VAK, partagent l'idée qu'une variété de modalités sensorielles de présentation du contenu pédagogique (le «multimédia ») contribue à maintenir l'intérêt et l'attention des apprenants. Ces auteurs ont toutefois précisé qu'il n'est pas nécessaire d'apparier le format de présentation du contenu pédagogique aux «styles d'apprentissage » VAK des apprenants.

De fait, varier les supports sensoriels n'est pas une pratique pédagogique dénuée de fondements scientifiques. Plusieurs recherches ont montré que la présentation bimodale de stimuli (p. ex. visuelle et auditive) procure un meilleur apprentissage qu'une présentation unimodale (Shams et Seitz, 2008). Macdonald et al. (2017) soulignent d'ailleurs que l'une des retombées plutôt inattendues de l'adhésion au neuromythe des styles d'apprentissage VAK est l'adoption de pratiques pédagogiques axées sur la présentation multisensorielle du contenu pédagogique. Selon ces auteurs, diversifier les modalités sensorielles de présentation du contenu pédagogique, même pour la mauvaise raison (la croyance envers les styles d'apprentissage VAK), pourrait donc s'avérer une pratique pédagogique efficace, appuyée par la recherche scientifique. Paradoxalement, selon eux, tenter de démystifier le neuromythe des styles d'apprentissage VAK chez les enseignants sans mentionner les travaux scientifiques qui appuient l'efficacité d'une présentation multisensorielle du contenu pédagogique pourrait malencontreusement les dissuader de continuer d'employer de telles méthodes efficaces pour l'ensemble des élèves. Puisque, selon Geake (2008), attribuer l'efficacité de méthodes didactiques multisensorielles à l'existence de styles d'apprentissage VAK pourrait consolider ce neuromythe, il serait nécessaire de trouver un moyen de convaincre les enseignants que de telles méthodes sont efficaces pour une raison autre que l'existence de styles d'apprentissage VAK chez leurs élèves.

Une piste intéressante en ce sens a été évoquée au tout début de cet article. La très grande majorité des recherches scientifiques en neuroéducation portent sur le fonctionnement du cerveau de l'apprenant en général. Comme l'ont souligné Arbuthnott et Krätzig (2015), les similitudes dans l'architecture cognitive du cerveau éclipsent considérablement les préférences individuelles d'apprentissage. Insister sur ces similitudes pourrait possiblement servir à convaincre les enseignants d'utiliser des pratiques pédagogiques générales, appuyées par la recherche scientifique, qui s'adressent à l'ensemble des élèves de leur classe (Agarwal et Roediger, 2018; Arbuthnott 
et Krätzig, 2015; Dunlosky et al., 2013). Or, comme nous l'avons évoqué d'entrée de jeu, le devoir des enseignants de répondre aux besoins individuels de leurs élèves pourrait les inciter à se détourner de pratiques pédagogiques générales probantes, au profit de pratiques inspirées de l'idée fausse de l'existence de profils particuliers de fonctionnement cérébral chez les apprenants. Ainsi, dans l'enquête québécoise récente réalisée par Blanchette Sarrasin et al. (2019), parmi les éléments ayant mené à leur niveau d'accord avec des énoncés faisant référence à des neuromythes populaires, les enseignants ont coché Cela correspond aux besoins de mes élèves dans une proportion de $38 \%$ (neuromythe des styles d'apprentissage VAK), de $30 \%$ (neuromythe des intelligences multiples) et de $12 \%$ (neuromythe de la dominance hémisphérique). Si l'on parvenait à convaincre les enseignants que des pratiques pédagogiques générales, qui s'adressent à l'ensemble des élèves de leur classe, contribuent également à répondre aux besoins individuels de leurs élèves, l'adoption de pratiques inspirées des neuromythes serait-elle moins fréquente?

Dans cet article, nous avons évoqué plusieurs mécanismes psychologiques qui semblent contribuer à l'adhésion aux neuromythes et à leur résistance face au savoir scientifique. Certains auteurs sont d'avis que l'introduction d'un cours sur la pensée critique dans la formation initiale en enseignement puisse possiblement réduire linfluence de tels mécanismes (Blanchette Sarrasin et al., 2019; Pasquinelli, 2012; Rousseau et al., 2018; Tardif et Doudin, 2011). Ce cours pourrait aborder, notamment, les procédés de validation non scientifiques (voir Larivée, 2001), les biais cognitifs (voir Gilovich, Griffin et Kahneman, 2002), ainsi que les raisonnements logiques fallacieux (voir Rousseau et al., 2018). Comme certains neuromythes seraient issus de découvertes scientifiques disqualifiées par la suite (p. ex. l'effet Mozart; voir Latendresse, Larivée et Miranda, 2006), un cours sur la pensée critique pourrait également comprendre des notions d'épistémologie. Enseigner aux apprentis enseignants que les découvertes scientifiques ne sont pas immuables, mais falsifiables (Popper, 1934/1973), de même que le concept de révolution scientifique (Kuhn, 1962/1983), pourrait possiblement contribuer à une conception moins « cristallisée » du savoir scientifique. Enfin, ce cours pourrait fournir des outils aux apprentis enseignants pour mieux distinguer entre savoir scientifique et savoir pseudoscientifique (Larivée, 2001; Lilienfeld, Ammirati et David, 2012).

En dernier lieu, nous aimerions soulever la possibilité que les avancées scientifiques en neuroéducation ne soient pas encore diffusées à une échelle suffisamment grande pour contribuer à l'adoption spontanée, dans la profession enseignante, de pratiques pédagogiques appuyées par la recherche. L'instauration de programmes universitaires en neuroéducation, à laquelle nous assistons depuis quelques années, a le potentiel de favoriser le transfert du savoir de la sphère scientifique à la sphère professionnelle. Pour les enseignants déjà en exercice, des tentatives louables de diffusion sont réalisées par le biais d'articles publiés dans des revues professionnelles (p. ex. Jenkins, 2017, 2018). Toutefois, une vigilance de tous les instants est requise pour débusquer, dans les revues professionnelles en éducation, des vestiges de neuromythes «classiques », ou encore des distorsions, extrapolations ou simplifications abusives de découvertes récentes en neuroéducation, qui seraient susceptibles de contribuer à la création de nouveaux neuromythes.

\section{Conclusion}

Les enquêtes internationales témoignent d'une adhésion massive aux neuromythes dans le milieu de l'éducation. Or, si certaines de ces fausses croyances sur le cerveau et l'apprentissage seraient issues d'une interprétation inexacte ou abusive de données expérimentales en neurosciences, la simple exposition aux neurosciences semble pourtant insuffisante pour les enrayer (p. ex. Im et al., 2018). Une telle exposition ne permettrait pas d'atteindre les fausses croyances, car celles-ci seraient profondément ancrées dans l'expérience personnelle des participants (Grospietsch et Mayer, 2018). En ce sens, une enquête récente réalisée auprès d'enseignants québécois a révélé que les répondants évoquent, plus que tout autre facteur, leurs intuitions ou leurs observations anecdotiques pour justifier leur adhésion à quatre neuromythes populaires sur cinq, dont celui des styles d'apprentissage VAK (Blanchette Sarrasin et al., 2019). De plus, dans une récente tentative de démystification réalisée auprès de professeurs britanniques, une fois confrontés au savoir scientifique disqualifiant l'utilité pédagogique des styles d'apprentissage, près du tiers des répondants ont manifesté de la résistance à abandonner ce concept dans leur pratique enseignante, dont $89 \%$ en raison de leur expérience personnelle (Newton et Miah, 2017).

Puisque les observations anecdotiques semblent alimenter les neuromythes et les protéger de l'assaut du savoir scientifique, dans la présente étude, nous avons proposé une intervention axée sur la création d'une anecdote personnelle disqualifiant l'utilité pédagogique du concept de styles d'apprentissage VAK. Notre hypothèse stipulait qu'une telle anecdote personnelle serait susceptible de former, avec le savoir scientifique, une alliance suffisamment puissante pour faire contrepoids aux anecdotes déjà vécues par les répondants et déstabiliser la fausse croyance envers l'utilité pédagogique de ce concept.

Des apprentis enseignants franco-ontariens inscrits en première année d'un programme de formation initiale à l'enseignement ont participé à une activité didactique démontrant l'absence de gains d'apprentissage lorsque le contenu pédagogique est présenté dans la modalité sensorielle correspondant à leur propre «style d'apprentissage » (présumé). Ces données personnelles ont ensuite été présentées de concert avec des données semblables issues de la littérature scientifique et démontrant également l'absence de gains d'apprentissage. À la suite de l'intervention, le pourcentage de participants indiquant vouloir malgré tout utiliser les styles d'apprentissage VAK dans leur pratique enseignante a décliné, passant de $100 \%$ à $60 \%$. 
Ce taux considérable de résistance à une intervention pourtant axée sur l'expérience personnelle des participants suggère que l'anecdote créée n'avait pas une puissance suffisante pour faire contrepoids aux anecdotes personnelles déjà vécues par les participants. Des pistes de recherche futures ont été suggérées pour consolider la nouvelle anecdote, dont notamment une intervention visant à réaliser l'activité didactique auprès d'élèves, de sorte que les participants puissent être témoins de contre-exemples directement issus du milieu éducatif.

\section{Remerciements}

Ce travail a bénéficié d'une subvention du Fonds des leaders de la Fondation canadienne pour l'innovation (FCI). Nous tenons à remercier les deux arbitres externes anonymes pour leurs critiques constructives de la version initiale de ce texte.

\section{Références}

Adey, P. et Dillon, J. (dir.). (2012). Bad education: Debunking myths in education. Berkshire, Royaume-Uni: Open University Press.

Agarwal, P. K. et Roediger, H. L. (2018). Lessons for learning: How cognitive psychology informs classroom practice. Phi Delta Kappan, 100(4), 8-12. https://doi.org/10.1177/0031721718815666

Alferink, L. A. (2007). Educational practices, superstitious behavior and mythed opportunities. Scientific Review of Mental Health Practice, 5(2), 21-30.

Álvarez-Montero, F. J., Reyes-Sosa, H., Leyva-Cruz, M. G. et Fragoza-Padilla, V. (soumis 2019). Learning styles and the human brain: What does the evidence tell us? Manuscrit soumis pour publication.

Arbuthnott, K. D. et Krätzig, G. P. (2015). Effective teaching: Sensory learning styles versus general memory processes. Comprehensive Psychology, 4.

https://doi.org/10.2466/06.IT.4.2

Aslaksen, K. et Lorås, H. (2018). The modality-specific learning style hypothesis: A mini-review. Frontiers in Psychology, 9. https://doi.org/10.3389/fpsyg.2018.01538

Aslaksen, K. et Lorås, H. (2019). Matching instruction with modality-specific learning style: Effects on immediate recall and working memory performance. Education Sciences, 9(1), 32. https://doi.org/10.3390/educsci9010032

Baillargeon, N. (2013). Légendes pédagogiques : L'autodéfense intellectuelle en éducation. Montréal, QC: Poètes de Brousse.
Barsch, J. (1991). Barsch Learning Style Inventory. Novato, CA : Academic Therapy.

Blanchette Sarrasin, J., Riopel, M. et Masson, S. (2019). Neuromyths and their origin among teachers in Quebec. Mind, Brain, and Education, 13(2), 100-109.

https://doi.org/10.1111/mbe.12193

Calvert, G. A., Campbell, R. et Brammer, M. J. (2000). Evidence from functional magnetic resonance imaging of crossmodal binding in the human heteromodal cortex. Current Biology, 10(11), 649-657. https://doi.org/10.1016/s0960-9822(00)00513-3

Cancela, J. M., Vila Suárez, M. H., Vasconcelos, J., Lima, A. et Ayán, C. (2015). Efficacy of Brain Gym training on the cognitive performance and fitness level of active older adults: A preliminary study. Journal of Aging and Physical Activity, 23(4), 653-658. https://doi.org/10.1123/japa.2014-0044

Chevrier, J., Fortin, G., Leblanc, R. et Théberge, M. (2000). Le style d'apprentissage: une perspective historique. Éducation et francophonie, 28(1), 20-46.

Coffield, F., Moseley, D., Hall, E. et Ecclestone, K. (2004a). Learning styles and pedagogy in post-16 learning: A systematic and critical review. Londres, Royaume-Uni : Learning and Skills Research Centre. http://hdl.voced.edu.au/10707/69027

Coffield, F., Moseley, D., Hall, E. et Ecclestone, K. (2004b). Should we be using learning styles? What research has to say to practice. Londres, Royaume-Uni : Learning and Skills Research Centre. http://hdl.voced.edu.au/10707/64981

Cuevas, J. et Dawson, B. L. (2018). A test of two alternatives cognitive processing models: Learning styles and dual coding. Theory and Research in Education, 16(1), 40-64. https://doi.org/10.1177/1477878517731450

Dandy, K. L. et Bendersky, K. (2014). Student and faculty beliefs about learning in higher education: Implications for teaching. International Journal of Teaching and Learning in Higher Education, 26(3), 358-380.

De Bruyckere, P., Kirschner, P. A. et Hulshof, C. D. (2015). Urban myths about learning and education. Amsterdam, Pays-Bas : Elsevier. https://doi.org/10.1016/C2013-0-18621-7

Dekker, S., Lee, N. C., Howard-Jones, P. et Jolles, J. (2012). Neuromyths in education: Prevalence and predictors of misconceptions among teachers. Frontiers in Psychology, 3. https://doi.org/10.3389/fpsyg.2012.00429

Dembo, M. H. et Howard, K. (2007). Advice about the use of learning styles: A major myth in education. Journal of College Reading and Learning, 37(2), 101-109. https://doi.org/10.1080/10790195.2007.10850200 
Dennison, P. E. et Dennison, G. E. (1994). Brain Gym ${ }^{\circledR}$ teacher's edition (éd. révisée). Ventura, CA : Edu-Kinesthetics.

Doudin, P.-A., Tardif, E. et Meylan, N. (2016). De l'utilité ambiguë des styles d'apprentissage et des neuromythes. Dans E. Tardif et P.-A. Doudin (dir.), Neurosciences et cognition: Perspectives pour les sciences de l'éducation (p. 81-102). Bruxelles, Belgique : De Boeck.

Dündar, S. et Gündüz, N. (2016). Misconceptions regarding the brain: The neuromyths of preservice teachers. Mind, Brain, and Education, 10(4), 212-232.

https://doi.org/10.1111/mbe.12119

Dunlosky, J., Rawson, K. A., Marsh, E. J., Nathan, M. J. et Willingham, D. T. (2013). Improving students' learning with effective learning techniques: Promising directions from cognitive and educational psychology. Psychological Science in the Public Interest, 14(1), 4-58.

https://doi.org/10.1177/1529100612453266

Feldman, J. (2016). The simplicity principle in perception and cognition. Wiley Interdisciplinary Reviews: Cognitive Science, 7(5), 330-340. https://doi.org/10.1002/wcs.1406

Ferrero, M., Garaizar, P. et Vadillo, M. A. (2016). Neuromyths in education: Prevalence among Spanish teachers and an exploration of cross-cultural variation. Frontiers in Human Neuroscience, 10. https://doi.org/10.3389/fnhum.2016.00496

Field, A. (2013). Discovering statistics using IBM SPSS Statistics ( $4^{\mathrm{e}}$ éd.). Thousand Oaks, CA : Sage.

Fleming, N. D. et Mills, C. (1992). Not another inventory, rather a catalyst for reflection. To Improve the Academy, 11(1), 137-155. https://doi.org/10.1002/j.2334-4822.1992.tb00213.x

Furnham, A. (2009). The validity of a new, self-report measure of multiple intelligence. Current Psychology, 28(4), 225239. https://doi.org/10.1007/s12144-009-9064-z

Gardner, H. (1983/1997). Frames of mind: A theory of multiple intelligences. New York, NY : Basic Books (traduit en 1997 sous le titre Les formes de l'intelligence. Paris, France : Odile Jacob).

Gardner, H. (2016). Multiple intelligences: Prelude, theory, and aftermath. Dans R. J. Sternberg, S. T. Fiske et D. J. Foss (dir.), Scientists making a difference (p. 167-170). New York, NY : Cambridge University Press.

Gazzaniga, M. S. (2005). Forty-five years of split-brain research and still going strong. Nature Reviews Neuroscience, 6(8), 653-659. https://doi.org/10.1038/nrn1723

Gazzaniga, M. S., Bogen, J. E. et Sperry, R. W. (1965). Observations on visual perception after disconnexion of the cerebral hemispheres in man. Brain, 88(2), 221-236.

https://doi.org/10.1093/brain/88.2.221
Geake, J. (2008). Neuromythologies in education. Educational Research, 50(2), 123-133. https://doi.org/10.1080/00131880802082518

Gilovich, T., Griffin, D. et Kahneman, D. (dir.). (2002). Heuristics and biases - The psychology of intuitive judgment. Cambridge, UK : Cambridge University Press. https://doi.org/10.1017/CBO9780511808098

Gleichgerrcht, E., Lira Luttges, B., Salvarezza, F. et Campos, A. L. (2015). Educational neuromyths among teachers in Latin America. Mind, Brain, and Education, 9(3), 170-178. https://doi.org/10.1111/mbe.12086

Grospietsch, F. et Mayer, J. (2018). Professionalizing preservice biology teachers' misconceptions about learning and the brain through conceptual change. Education Sciences, 8(3), 120. https://doi.org/10.3390/educsci8030120

Grospietsch, F. et Mayer, J. (2019). Pre-service science teachers' neuroscience literacy: Neuromyths and a professional understanding of learning and memory. Frontiers in Human Neuroscience, 13. https://doi.org/10.3389/fnhum.2019.00020

Hermida, M. J., Segretin, M. S., García, A. S. et Lipina, S. J. (2016). Conceptions and misconceptions in preschool teachers: A study from Argentina. Educational Research, 58(4), 457-472. https://doi.org/10.1080/00131881.2016.1238585

Holland, S. K., Vannest, J., Mecoli, M., Jacola, L. M., Tillema, J.M., Karunanayaka, P. R., ... et Byars, A. W. (2007). Functional MRI of language lateralization during development in children. International Journal of Audiology, 46(9), 533-551. https://doi.org/10.1080/14992020701448994

Hood, B., Howard-Jones, P. A, Laurillard, D., Bishop, D., Coffield, F., Frith, U., ... et Foulsham, T. (2017, 12 mars). No evidence to back idea of learning styles. The Guardian. https://www.theguardian.com/education/2017/mar/1 2/no-evidence-to-back-idea-of-learning-styles

Howard-Jones, P. A. (2014). Neuroscience and education: Myths and messages. Nature Reviews Neuroscience, 15(12), 817-824. https://doi.org/10.1038/nrn3817

Howard-Jones, P. A., Franey, L., Mashmoushi, R. et Liao, Y.-C. (2009, septembre). The neuroscience literacy of trainee teachers. Dans British Educational Research Association Annual Conference (p. 1-39). Manchester, Royaume-Uni : University of Manchester.

Husmann, P. R. et O'Loughlin, V. D. (2019). Another nail in the coffin for learning styles? Disparities among undergraduate anatomy students' study strategies, class performance, and reported VARK learning styles. Anatomical Sciences Education, 12(1), 6-19. https://doi.org/10.1002/ase.1777 
Hyatt, K. J. (2007). Brain Gym ${ }^{\circledR}$ : Building stronger brains or wishful thinking? Remedial and Special Education, 28(2), 117-124. https://doi.org/10.1177/07419325070280020201

Im, S.-h., Cho, J.-Y., Dubinsky, J. M. et Varma, S. (2018). Taking an educational psychology course improves neuroscience literacy but does not reduce belief in neuromyths. PLoS ONE, 13(2), e0192163.

https://doi.org/10.1371/journal.pone.0192163

Jenkins, R. T. (2017). Using educational neuroscience and psychology to teach science. Part 1 : A case study review of Cognitive Load Theory (CLT) and Cognitive Acceleration through Science Education (CASE). School Science Review, 99(367), 93-103.

Jenkins, R. T. (2018). Using educational neuroscience and psychology to teach science. Part 2 . A case study review of 'The Brain-Targeted Teaching Model' and 'ResearchBased Strategies to Ignite Student Learning'. School Science Review, 100(371), 66-75.

Karakus, O., Howard-Jones, P. A. et Jay, T. (2015). Primary and secondary school teachers' knowledge and misconceptions about the brain in Turkey. Procedia - Social and Behavioral Sciences, 174, 1933-1940.

https://doi.org/10.1016/j.sbspro.2015.01.858

Kim, M. et Sankey, D. (2018). Philosophy, neuroscience, and pre-service teachers' beliefs in neuromyths: A call for remedial action. Educational Philosophy and Theory, 50(13), 1214-1227.

https://doi.org/10.1080/00131857.2017.1395736

Kirby, J. R., Moore, P. J. et Schofield, N. J. (1988). Verbal and visual learning styles. Contemporary Educational Psychology, 13(2), 169-184.

https://doi.org/10.1016/0361-476X(88)90017-3

Kirschner, P. A. (2017). Stop propagating the learning styles myth. Computers and Education, 106, 166-171.

https://doi.org/10.1016/j.compedu.2016.12.006

Knoll, A. R., Otani, H., Skeel, R. L. et Van Horn, K. R. (2017). Learning style, judgements of learning, and learning of verbal and visual information. British Journal of Psychology, 108(3), 544-563. https://doi.org/10.1111/bjop.12214

Kolb, D. A. (1976). LSI - The Learning Style Inventory: Technical manual. Boston, MA : McBer.

Kozhevnikov, M., Evans, C. et Kosslyn, S. M. (2014). Cognitive style as environmentally sensitive individual differences in cognition: A modern synthesis and applications in education, business, and management. Psychological Science in the Public Interest, 15(1), 3-33.

https://doi.org/10.1177/1529100614525555
Krätzig, G. P. et Arbuthnott, K. D. (2006). Perceptual learning style and learning proficiency: A test of the hypothesis. Journal of Educational Psychology, 98(1), 238-246. https://doi.org/10.1037/0022-0663.98.1.238

Kuhn, T. S. (1962/1983). La structure des révolutions scientifiques. Paris, France : Flammarion.

Larivée, S. (2001). Science contre pseudosciences : un combat inégal. Revue canadienne de psycho-éducation, 30(1), 1-25.

Larivée, S., Baribeau, J. et Pflieger, J.-F. (2008). Qui utilise $10 \%$ de son cerveau? Revue de psychoéducation, 37(1), 117-142.

Larivée, S. et Sénéchal, C. (2012). Que dit la science à propos des intelligences multiples? Revue québécoise de psychologie, 33(1), 23-45.

Latendresse, C., Larivée, S. et Miranda, D. (2006). La portée de l'effet Mozart: Succès souvenirs, fausses notes et reprises. Psychologie canadienne, 47(2), 125-142. https://doi.org/10.1037/cp2006004

Leite, W. L., Svinicki, M. et Shi, Y. (2010). Attempted validation of the scores of the VARK: Learning Styles Inventory with multitrait-multimethod confirmatory factor analysis models. Educational and Psychological Measurement, 70(2), 323-339. https://doi.org/10.1177/0013164409344507

Lilienfeld, S. O., Ammirati, R. et David, M. (2012). Distinguishing science from pseudoscience in school psychology: Science and scientific thinking as safeguards against human error. Journal of School Psychology, 50(1), 7-36. https://doi.org/10.1016/j.jsp.2011.09.006

Lindell, A. K. et Kidd, E. (2011). Why right-brain teaching is half-witted: A critique of the misapplication of neuroscience to education. Mind, Brain, and Education, 5(3), 121-127. https://doi.org/10.1111/j.1751-228x.2011.01120.x

Loi Zedda, M. (2014). Croyances chez les enseignants $d u$ primaire et du spécialisé à l'égard des neuromythes. Étude comparée entre la Suisse Romande et le Québec. Mémoire de master inédit, Haute École Pédagogique, Lausanne, Suisse.

Macdonald, K., Germine, L., Anderson, A., Christodoulou, J. et McGrath, L. M. (2017). Dispelling the myth: Training in education or neuroscience decreases but does not eliminate beliefs in neuromyths. Frontiers in Psychology, 8. https://doi.org/10.3389/fpsyg.2017.01314

Masson, S. (2015). Les apports de la neuroéducation à l'enseignement: des neuromythes aux découvertes actuelles. Approche neuropsychologique des apprentissages chez l'enfant, 134, 11-22. 
Newton, P. M. (2015). The learning styles myth is thriving in higher education. Frontiers in Psychology, 6. https://doi.org/10.3389/fpsyg.2015.01908

Newton, P. M. et Miah, M. (2017). Evidence-based higher education: Is the learning styles 'myth' important? Frontiers in Psychology, 8. https://doi.org/10.3389/fpsyg.2017.00444

Nielsen, J. A., Zielinski, B. A., Ferguson, M. A., Lainhart, J. E. et Anderson, J. S. (2013). An evaluation of the left-brain vs. right-brain hypothesis with resting state functional connectivity magnetic resonance imaging. PLoS ONE,8(8), e71275. https://doi.org/10.1371/journal.pone.0071275

Nyhan, B. et Reifler, J. (2010). When corrections fail: The persistence of political misperceptions. Political Behavior, 32(2), 303-330. https://doi.org/10.1007/s11109-010-9112-2

Othman, N. et Amiruddin, M. H. (2010). Different perspectives of learning styles from VARK model. Procedia - Social and Behavioral Sciences, 7, 652-660.

https://doi.org/10.1016/j.sbspro.2010.10.088

Paivio, A. (1971). Imagery and verbal processes. New York, NY : Holt, Rinehart, and Winston.

Paivio, A. (1986). Mental representations: A dual coding approach. Oxford, Royaume-Uni : Oxford University Press.

Papadatou-Pastou, M., Gritzali, M. et Barrable, A. (2018). The learning styles educational neuromyth: Lack of agreement between teachers' judgments, selfassessment, and students' intelligence. Frontiers in Education, 3. https://doi.org/10.3389/feduc.2018.00105

Papadatou-Pastou, M., Haliou, E. et Vlachos, F. (2017). Brain knowledge and prevalence of neuromyths among prospective teachers in Greece. Frontiers in Psychology, 8. https://doi.org/10.3389/fpsyg.2017.00804

Pashler, H., McDaniel, M., Rohrer, D. et Bjork, R. (2008). Learning styles: Concepts and evidence. Psychological Science in the Public Interest, 9(3), 105-119. https://doi.org/10.1111/j.1539-6053.2009.01038.x

Pasquinelli, E. (2012). Neuromyths: Why do they exist and persist? Brain, Mind, and Education, 6(2), 89-96. https://doi.org/10.1111/j.1751-228x.2012.01141.x

Pasquinelli, E. (2015). Mon cerveau, ce héros: Mythes et réalités. Paris, France : Le Pommier.

Popper, K. R. (1934/1973). La logique de la découverte scientifique. Lausanne, Suisse : Payot.

Riener, C. et Willingham, D. (2010). The myth of learning styles. Change: The Magazine of Higher Learning, 42(5), 32-35. https://doi.org/10.1080/00091383.2010.503139
Risen, J. et Gilovich, T. (2007). Informal logical fallacies. Dans R. J. Sternberg, H. L. Roediger III et D. F. Halpern (dir.), Critical thinking in psychology (p. 110-130). New York, NY : Cambridge University Press. https://doi.org/10.1017/cbo9780511804632.008

Rogowsky, B. A., Calhoun, B. M. et Tallal, P. (2015). Matching learning style to instructional method: Effects on comprehension. Journal of Educational Psychology, 107(1), 64-78. https://doi.org/10.1037/a0037478

Rogowsky, B. A., Calhoun, B. M. et Tallal, P. (2020). Providing instruction based on students' learning style preferences does not improve learning. Frontiers in Psychology, 11. https://doi.org/10.3389/fpsyg.2020.00164

Rohrer, D. et Pashler, H. (2012). Learning styles: Where's the evidence? Medical Education, 46(7), 634-635. https://doi.org/10.1111/j.1365-2923.2012.04273.x

Rousseau, L., Gauthier, Y. et Caron, J. (2018). L'utilité des «styles d'apprentissage » VAK (auditif, visuel, kinesthésique) en éducation: entre l'hypothèse de recherche et le mythe scientifique. Revue de psychoéducation, 47(2), 409-448. https://doi.org/10.7202/1054067ar

Ruhaak, A. E. et Cook, B. G. (2018). The prevalence of educational neuromyths among pre-service special education teachers. Mind, Brain, and Education, 12(3), 155-161. https://doi.org/10.1111/mbe.12181

Schacter, D. L., Harbluk, J. L. et McLachlan, D. R. (1984). Retrieval without recollection: An experimental analysis of source amnesia. Journal of Verbal Learning and Verbal Behavior, 23(5), 593-611. https://doi.org/10.1016/s0022-5371(84)90373-6

Scott, C. (2010). The enduring appeal of 'learning styles'. Australian Journal of Education, 54(1), 5-17. https://doi.org/10.1177/000494411005400102

Shams, L. et Seitz, A. R. (2008). Benefits of multisensory learning. Trends in Cognitive Sciences, 12(11), 411-417. https://doi.org/10.1016/j.tics.2008.07.006

Sharp, J. G., Bowker, R. et Byrne, J. (2008). VAK or VAK-uous? Towards the trivialisation of learning and the death of scholarship. Research Papers in Education, 23(3), 293-314. https://doi.org/10.1080/02671520701755416

Spaulding, L. S., Mostert, M. P. et Beam, A. P. (2010). Is Brain $\mathrm{Gym}^{\circledR}$ an effective educational intervention? Exceptionality, 18(1), 18-30. https://doi.org/10.1080/09362830903462508

Sperry, R. (1982). Some effects of disconnecting the cerebral hemispheres. Science, 217(4566), 1223-1226. https://doi.org/10.1126/science.7112125

Stahl, S. A. (1999). Different strokes for different folks? A critique of learning styles. American Educator, 23(3), 27-31. 
Stephenson, J. (2009). Best practice? Advice provided to teachers about the use of Brain $\mathrm{Gym}^{\circledR}$ in Australian schools. Australian Journal of Education, 53(2), 109-124. https://doi.org/10.1177/000494410905300202

Tardif, E. et Doudin, P.-A. (2010). Neurosciences, neuromythes et sciences de l'éducation. Prismes : revue pédagogique HEP Vaud, 12, 11-14.

http://hdl.handle.net/20.500.12162/1013

Tardif, E. et Doudin, P.-A. (2011). Neurosciences cognitives et éducation: le début d'une collaboration. Formation et pratiques d'enseignement en questions, 12, 95-116.

Tardif, E., Doudin, P.-A. et Meylan, N. (2015). Neuromyths among teachers and student teachers. Mind, Brain, and Education, 9(1), 50-59. https://doi.org/10.1111/mbe.12070

Tversky, A. et Kahneman, D. (1973). Availability: A heuristic for judging frequency and probability. Cognitive Psychology, 5(2), 207-232.

https://doi.org/10.1016/0010-0285(73)90033-9
Visser, B. A., Ashton, M. C. et Vernon, P. A. (2006). Beyond g: Putting multiple intelligences theory to the test. Intelligence, 34(5), 487-502. https://doi.org/10.1016/j.intell.2006.02.004

Waterhouse, L. (2006). Multiple intelligences, the Mozart effect, and emotional intelligence: A critical review. Educational Psychologist, 41(4), 207-225. https://doi.org/10.1207/s15326985ep4104_1

Willingham, D. T. (2004). Reframing the mind - Howard Gardner became a hero among educators simply by redefining talents as "intelligences". Education Next, 4(3), 19-24.

Willingham, D. T., Hughes, E. M. et Dobolyi, D. G. (2015). The scientific status of learning styles theories. Teaching of Psychology, 42(3), 266-271. https://doi.org/10.1177/0098628315589505

Zhang, R., Jiang, Y., Dang, B. et Zhou, A. (2019). Neuromyths in Chinese classrooms: Evidence from headmasters in an underdeveloped region in China. Frontiers in Education, 4. https://doi.org/10.3389/feduc.2019.00008 